\title{
Photoinduced 1,4-Additions of Indoles to Enones
}

\author{
Joseph Moran, Tanya Suen, and André M. Beauchemin* \\ Department of Chemistry, University of Ottawa, 10 Marie Curie, Ottawa, Ontario, Canada, K1N 6N5 \\ andre.beauchemin@uottawa.ca \\ Supporting Information
}

Table of Contents

page

General Information $\quad$ S1

$\begin{array}{ll}\text { Materials } & \text { S2 }\end{array}$

General Procedure for Solvent Scan (Table 1) S2

Table 5. Concentration Scan for the Photoinduced 1,4-Addition of Indole to Cyclohept-2-enone S2

General Procedure for Concentration Scan (Table 5) $\quad$ S2

General Procedure for Photoinduced 1,4-Addition of Indoles (Tables 2, 3 and 4). S3

Table 2a. Experimental Details for Results in Table $2 \quad$ S4

Table 3a. Experimental Details for Results in Table $3 \quad$ S5

Table 4a. Experimental Details for Results in Table $4 \quad$ S8

Procedure for Attempted Low Temperature Generation/Trapping of (E)-cyclooct-2-enone. S9

$\begin{array}{lr}\text { Procedure for Oxygen-Induced Quenching of Enone Triplet. } & \text { S10 }\end{array}$

$\begin{array}{lr}\text { Discussion of Mechanistic Alternatives } & \text { S10 }\end{array}$

$\begin{array}{lr}\text { NMR Spectra } & \text { S12 }\end{array}$

General Information. All reactions were performed in air-dried or oven-dried borosilicate tubes. Reactions were either carried out in a Rayonet photoreactor equipped with eight $8 \mathrm{~W}$ ultraviolet lamps or between two Luzchem exposure panels equipped with four $8 \mathrm{~W}$ ultraviolet lamps each. UV-A bulbs $(\sim 350$ $\mathrm{nm}),{ }^{1,2} \mathrm{UV}-\mathrm{B}$ bulbs $(\sim 300 \mathrm{~nm})$, and exposure panels were purchased from Luzchem Research, Inc. Purification of reaction products was carried out by flash column chromatography using silica gel (40-63 $\mu \mathrm{m})$. Analytical thin layer chromatography was performed on aluminum sheets pre-coated with silica gel 60 F254, cut to size. Visualization was accomplished with UV light and an aqueous vanillin solution followed by heating.

${ }^{1} \mathrm{H}$ NMR spectra were recorded on a $300 \mathrm{MHz}$ spectrometer at ambient temperature and are reported in ppm using solvent as the internal standard $\left(\mathrm{CDCl}_{3}\right.$ at $7.26 \mathrm{ppm}$ or $\mathrm{C}_{6} \mathrm{D}_{6}$ at $\left.7.15 \mathrm{ppm}\right)$. Data are reported as: multiplicity ( $\mathrm{ap}=$ apparent, $\mathrm{br}=$ broad, $\mathrm{s}=$ singlet, $\mathrm{d}=$ doublet, $\mathrm{t}=$ triplet, $\mathrm{q}=$ quartet, $\mathrm{m}=$ multiplet), integration and coupling constant(s) in $\mathrm{Hz} .{ }^{13} \mathrm{C}$ NMR spectra were recorded at $75 \mathrm{MHz}$. Chemical shifts are reported in ppm from tetramethylsilane, with the solvent resonance employed as the internal standard $\left(\mathrm{CDCl}_{3}\right.$ at $77.0 \mathrm{ppm}$ or $\mathrm{C}_{6} \mathrm{D}_{6}$ at $\left.128.02 \mathrm{ppm}\right)$. High-resolution mass spectra were obtained from the Ottawa-Carleton Mass Spectrometry Centre.

(1) For specifications, see http://www.luzchem.com/handbook/LESUVA011.pdf

(2) Alternatively, this apparatus could be substituted for a medium pressure Hg lamp fitted with a uranium glass filter. 
Materials. Dichloromethane was dried by distillation over calcium hydride. Tetrahydrofuran was dried by distillation over sodium/benzophenone ketyl. Diethyl ether was purified by passage through a column of activated alumina under a nitrogen atmosphere. ${ }^{3}$ Unless otherwise noted, all commercial materials were used without further purification. Cyclohept-2-enone and cyclooct-2-enone were prepared according to literature procedures. $^{4,5}$ 1-Benzylindole and 1-allylindole were prepared according to the procedure of Guida. ${ }^{6}$ 1Acetylindole was prepared according to the procedure of Nickisch. ${ }^{7}$ tert-Butylindole-1-carboxylate was prepared according to the procedure of Vazquez. ${ }^{8}$

General Procedure for Solvent Scan (Table 1). Into each of 10 borosilicate tubes equipped with stirbars was added cyclohept-2-enone $(0.033 \mathrm{~g}, 0.30 \mathrm{mmol})$ and indole $(0.053 \mathrm{~g}, 0.45 \mathrm{mmol})$. To each tube was added $2 \mathrm{~mL}$ of solvent (Table 1). In the case of volatile solvents, the volume was marked on the tube, and an excess of solvent was added. The tube was capped with a rubber septum and purged with a nitrogen balloon and an outlet while stirring for a minimum of 5 minutes or until the solvent had evaporated to the marked level. The tubes were then placed on a Luzchem rotating carousel equipped with a stirplate and allowed to stir in a Rayonet photoreactor equipped with eight $8 \mathrm{~W}$ UV-A bulbs for 18 hours. The reactions were monitored by TLC (40\% EtOAc/hexanes). The contents of each tube were then transferred to a round-bottom flask, and the solvent was removed under reduced pressure. To each flask was added $1.00 \mathrm{~mL}$ of a standardized solution of $1 \%$ tetramethylsilane in deuterochloroform. ${ }^{1} \mathrm{H}$ NMR spectra of these solutions were recorded, and the \% conversion calculated based on the ratio of the tetramethysilane $(12 \mathrm{H})$ resonance at $0.0 \mathrm{ppm}$ to the resonance corresponding to the product's methine proton $(1 \mathrm{H})$ at $3.26 \mathrm{ppm}$. The method is estimated to have an error of approximately $\pm 5 \%$.

Table 5. Concentration Scan for the Photoinduced 1,4-Addition of Indole to Cyclohept-2-enone

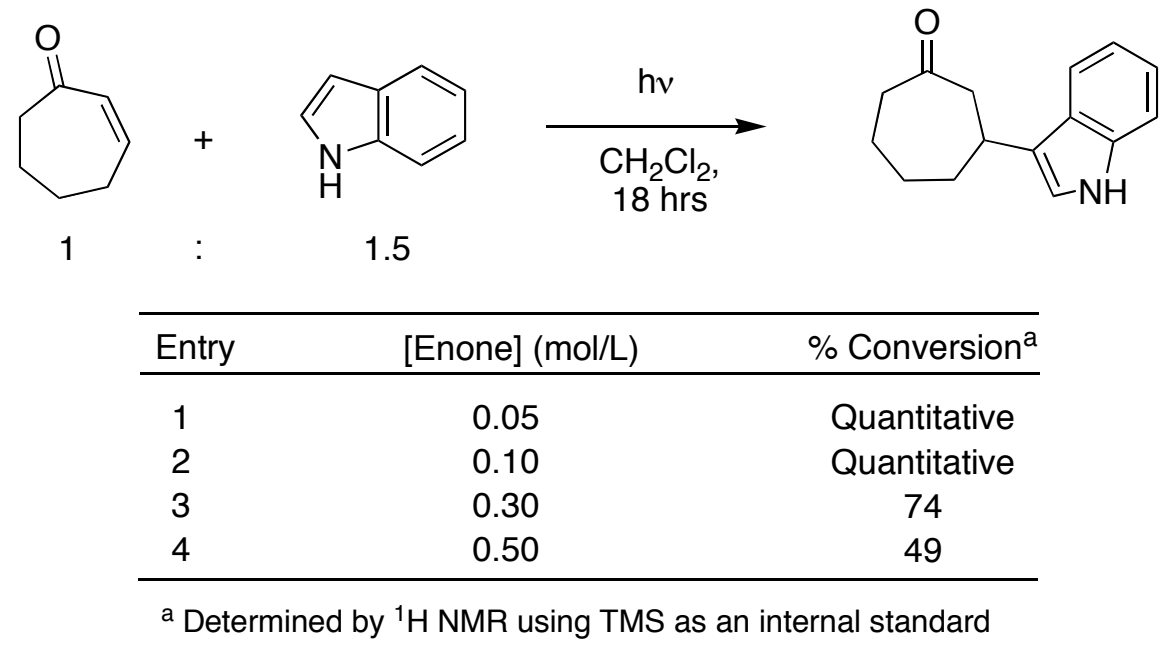

General Procedure for Concentration Scan (Table 5). Into each of 4 borosilicate tubes equipped with stirbars were added cyclohept-2-enone $(0.033 \mathrm{~g}, 0.30 \mathrm{mmol})$ and indole $(0.053 \mathrm{~g}, 0.45 \mathrm{mmol})$. To each tube

(3) Pangborn, A. B.; Giardello, M. A.; Grubbs, R. H.; Rosen, R. K.; Timmers, F. J. Organometallics 1996, $15,1518$.

(4) Mihelich, E. D.; Eickhoff, D. J. J. Org. Chem. 1983, 48, 4135.

(5) Chidambaram, N.; Chandrasekaran, S. J. Org. Chem. 1987, 52, 5048.

(6) Guida, W. C.; Mathre, D. J. J. Org. Chem. 1980, 45, 3172.

(7) Nickisch, K.; Klose, W.; Bohlmann, F. Chem. Ber. 1980, 113, 2036.

(8) Vazquez, E.; Davies, I. W.; Payack, J. F. J. Org. Chem. 2002, 67, 7551. 
was then added either $6.0,3.0,1.0$, or $0.60 \mathrm{~mL}$ of dichloromethane to give concentrations of $0.050,0.10,0.30$ and $0.50 \mathrm{M}$, respectively. The volume was marked on the tube, and an excess of solvent was added. The tube was capped with a rubber septum and purged with a nitrogen balloon and an outlet while stirring for a minimum of 5 minutes or until the solvent had evaporated to the marked level. The tubes were then placed on a Luzchem rotating carousel equipped with a stirplate and allowed to stir in a Rayonet photoreactor equipped with eight $8 \mathrm{~W}$ UV-A bulbs for 18 hours. The contents of each tube were then transferred to a round-bottom flask, and the solvent was removed under reduced pressure. To each flask was added $1.00 \mathrm{~mL}$ of a standardized solution of $1 \%$ tetramethylsilane in deuterochloroform. ${ }^{1} \mathrm{H}$ NMR spectra of these solutions were recorded, and the $\%$ conversion calculated based on the ratio of the tetramethysilane $(12 \mathrm{H})$ resonance at $0.0 \mathrm{ppm}$ to the resonance corresponding to the product's methine proton $(1 \mathrm{H})$ at $3.26 \mathrm{ppm}$. The method is estimated to have an error of approximately $\pm 5 \%$.

General Procedure for Photoinduced 1,4-Addition of Indoles (Tables 2, 3 and 4).

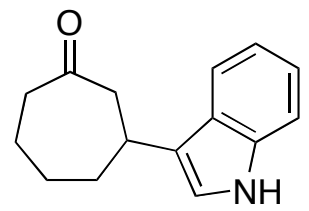

3-(1H-Indol-3-yl)cycloheptanone (3a)(table 2, entry 4). A borosilicate tube was charged with a stir bar, cyclohept-2-enone $(0.105 \mathrm{~g}, 0.949 \mathrm{mmol})$ and indole $(0.117 \mathrm{~g}, 0.999 \mathrm{mmol})$. A volume of $18.0 \mathrm{~mL}$ dichloromethane was added to the mixture and the volume was marked on the tube. An excess of 1-2 mL of solvent was then added to the tube. The tube was capped with a septum and was purged with stirring using a nitrogen balloon and an outlet until the solvent evaporated to the volume marked. The reaction mixture was then placed on a stir-plate and allowed to stir between two exposure panels each equipped with four 8W UV-A bulbs for 18 hours to obtain the crude mixture as a dark red liquid. ${ }^{9}$ The reaction was monitored by TLC and the product was found to have TLC $R_{\mathrm{f}} 0.51$ on silica gel (40\% EtOAc/hexanes). The crude mixture was concentrated under reduced pressure to a few milliliters and was purified by flash chromatography using $\mathrm{CH}_{2} \mathrm{Cl}_{2}$. The purified product was a clear, colorless oil $(0.211 \mathrm{~g}, 98 \%$ yield $) ;{ }^{1} \mathrm{H} \mathrm{NMR}\left(\mathrm{C}_{6} \mathrm{D}_{6}, 300 \mathrm{MHz}\right) \delta 7.65-$ $7.62(\mathrm{~m}, 1 \mathrm{H}), 7.26-7.17(\mathrm{~m}, 2 \mathrm{H}), 7.12-7.09(\mathrm{~m}, 1 \mathrm{H}), 6.99(\mathrm{br} \mathrm{s}, 1 \mathrm{H}), 6.38(\mathrm{~d}, 1 \mathrm{H}, J=2.4 \mathrm{~Hz}), 3.05(\mathrm{tt}, 1 \mathrm{H}, J=$ 10.4 and $2.7 \mathrm{~Hz}), 2.79(\mathrm{ddd}, 1 \mathrm{H}, J=14.4,3.6$ and $1.5 \mathrm{~Hz}), 2.71(\mathrm{dd}, 1 \mathrm{H}, J=14.4$ and $10.5 \mathrm{~Hz}), 2.29($ ap dd, $2 \mathrm{H}$, $J=9.2$ and $4.5 \mathrm{~Hz}), 2.07-2.01(\mathrm{~m}, 1 \mathrm{H}), 1.63-1.43(\mathrm{~m}, 3 \mathrm{H}), 1.36-1.09(\mathrm{~m}, 2 \mathrm{H}) ;{ }^{13} \mathrm{C} \mathrm{NMR}\left(\mathrm{C}_{6} \mathrm{D}_{6}, 75 \mathrm{MHz}\right) \delta$ 211.7, 136.9, 126.5, 122.2, 121.4, 119.6, 119.5, 119.4, 111.5, 50.5, 43.8, 38.2, 34.0, 29.2, 24.4; IR (film): 3411, 3057, 2927, 2856, 1691, 1458, 1339, 1099, $741 \mathrm{~cm}^{-1}$; HRMS (EI): Exact mass calcd for $\mathrm{C}_{15} \mathrm{H}_{17} \mathrm{NO}[\mathrm{M}]^{+}$: 227.1310. Found: 227.1304.

(9) The described transformations were typically accompanied by a change in color from a clear to a red or an amber solution. 
Table 2a. Experimental Details for Results in Table 2

\begin{tabular}{cccccc}
\hline Entry & $\mathrm{m}_{\text {enone }}(\mathrm{g})$ & $\mathrm{n}_{\text {enone }}(\mathrm{mmol})$ & $\mathrm{m}_{\text {indole }}(\mathrm{g})$ & $\mathrm{n}_{\text {indole }}(\mathrm{mmol})$ & $\mathrm{v}_{\text {solvent }}(\mathrm{mL})$ \\
\hline 1 & 0.034 & 0.49 & 0.080 & 0.68 & 10 \\
2 & 0.033 & 0.40 & 0.071 & 0.61 & 8.0 \\
3 & 0.033 & 0.34 & 0.060 & 0.51 & 7.0 \\
4 & 0.105 & 0.949 & 0.117 & 0.999 & 19 \\
5 & 0.033 & 0.27 & 0.047 & 0.40 & 5.5 \\
\hline
\end{tabular}

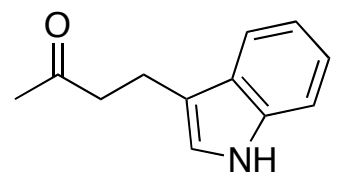

4-(1H-Indol-3-yl)butan-2-one) (5)(table 2, entry 1). Isolated $42 \mathrm{mg}$ as white crystals after column chromatography $(15 \%$ EtOAc/hexanes). Spectral data were found to be in good agreement with those in the literature. $^{10}$<smiles>O=C1CCC(c2c[nH]c3ccccc23)C1</smiles>

3-(1H-Indol-3-yl)cyclopentanone (7)(table 2, entry 2). Isolated $15 \mathrm{mg}$ as a white powder after column chromatography $(25 \%$ EtOAc/hexanes). Spectral data were found to be in good agreement with those in the literature. $^{11}$<smiles>O=C1CCCC(c2c[nH]c3ccccc23)C1</smiles>

3-(1H-Indol-3-yl)cyclohexanone (9)(table 2, entry 3). Isolated $10 \mathrm{mg}$ as a clear, colorless oil after column chromatography (30\% EtOAc/hexanes). Spectral data were found to be in good agreement with those in the literature. $^{12}$

(10) Yadav, J. S.; Abraham, S.; Reddy, B. V. S.; Sabitha, G. Synthesis 2001, 14, 2165.

(11) Bandini, M.; Cozzi, P. G.; Giacomini, M.; Melchiorre, P.; Selva, S.; Umani-Ronchi, A. J. Org. Chem. 2002, 67, 3700.

(12) Magnus, P.; Lacour, J.; Evans, P. A.; Rigollier, P.; Tobler, H. J. Am. Chem. Soc. 1998, 120, 12486. 


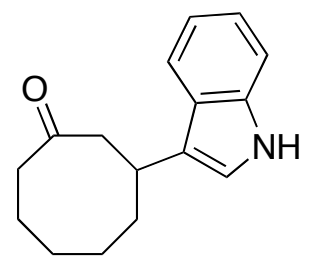

3-(1H-Indol-3-yl)cyclooctanone (11)(table 2, entry 5). Isolated $43 \mathrm{mg}$ as a clear, colorless oil after column chromatography (20\% EtOAc/hexanes). TLC $R_{\mathrm{f}} 0.53$ (40\% EtOAc/hexanes); ${ }^{1} \mathrm{H}$ NMR $\left(\mathrm{CDCl}_{3}, 300 \mathrm{MHz}\right) \delta$ 7.96 (br s, $1 \mathrm{H}), 7.68(\mathrm{~d}, 1 \mathrm{H}, J=7.8 \mathrm{~Hz}), 7.36(\mathrm{~d}, 1 \mathrm{H}, J=8.1 \mathrm{~Hz}), 7.19(\mathrm{td}, 1 \mathrm{H}, J=7.2$ and $1.2 \mathrm{~Hz}) .7 .12(\mathrm{td}$, $1 \mathrm{H}, J=7.8$ and $0.9 \mathrm{~Hz}), 6.98(\mathrm{~d}, 1 \mathrm{H}, J=2.4 \mathrm{~Hz}), 3.57(\mathrm{tt}, 1 \mathrm{H}, J=12$ and $3.3 \mathrm{~Hz}), 2.92(\mathrm{t}, 1 \mathrm{H}, J=12.6 \mathrm{~Hz}), 2.66$ (ddd, $1 \mathrm{H}, J=12.9,3.2$ and $1.2 \mathrm{~Hz}), 2.58(\mathrm{dd}, 1 \mathrm{H}, J=12.3$ and $3 \mathrm{~Hz}), 2.46-2.39(\mathrm{~m}, 1 \mathrm{H}), 2.18-1.42(\mathrm{~m}, 8 \mathrm{H})$. ${ }^{13} \mathrm{C} \mathrm{NMR}\left(\mathrm{CDCl}_{3}, 75 \mathrm{MHz}\right) \delta 213.9,136.9,126.8,122.4,121.6,119.9,119.7,119.6,111.6,48.3,42.8,35.7$, 34.5, 27.9, 25.1, 24.6; IR (film): 3410, 3055, 2929, 2857, 1686, 1458, 1339, $740 \mathrm{~cm}^{-1}$; HRMS (EI): Exact mass calcd for $\mathrm{C}_{16} \mathrm{H}_{19} \mathrm{NO}[\mathrm{M}]^{+}:$241.1467. Found: 241.1487 .

Table 3a. Experimental Details for Results in Table 3

\begin{tabular}{cccccc}
\hline Entry & $\mathrm{m}_{\text {enone }}(\mathrm{g})$ & $\mathrm{n}_{\text {enone }}(\mathrm{mmol})$ & $\mathrm{m}_{\text {indole }}(\mathrm{g})$ & $\mathrm{n}_{\text {indole }}(\mathrm{mmol})$ & $\mathrm{V}_{\text {solvent }}(\mathrm{mL})$ \\
\hline 1 & 0.105 & 0.949 & 0.117 & 0.999 & 19 \\
2 & 0.100 & 0.907 & 0.131 & 0.998 & 18 \\
3 & 0.050 & 0.45 & 0.263 & 1.36 & 9.0 \\
4 & 0.033 & 0.30 & 0.132 & 0.906 & 6.0 \\
5 & 0.050 & 0.45 & 0.178 & 1.36 & 9.0 \\
6 & 0.050 & 0.45 & 0.267 & 1.36 & 9.0 \\
7 & 0.050 & 0.45 & 0.184 & 1.36 & 9.0 \\
8 & 0.050 & 0.45 & 0.239 & 1.36 & 9.0 \\
9 & 0.050 & 0.45 & 0.193 & 1.36 & 9.0 \\
10 & 0.050 & 0.45 & 0.221 & 1.36 & 9.0 \\
\hline
\end{tabular}<smiles>Cc1[nH]c2ccccc2c1C1CCCCC(=O)C1</smiles>

3-(2-Methyl-1H-indol-3-yl)cycloheptanone (3b)(table 3, entry 2). Isolated $128 \mathrm{mg}$ as a clear, colorless oil after column chromatography $\left(\mathrm{CH}_{2} \mathrm{Cl}_{2}\right)$. TLC $R_{\mathrm{f}} 0.35\left(25 \%\right.$ EtOAc/hexanes); ${ }^{1} \mathrm{H}$ NMR $\left(\mathrm{C}_{6} \mathrm{D}_{6}, 300 \mathrm{MHz}\right) \delta$ 7.60-7.57 (m, 1H), 7.21 (ddd, 2H, $J=13.9,7.0$, and 1.5 Hz), 7.07-7.04 (m, 1H), 6.46 (br s, 1H), 3.14 (dd, $1 \mathrm{H}, J$ $=13.4$ and $12.1 \mathrm{~Hz}), 3.05-2.95(\mathrm{~m}, 1 \mathrm{H}), 2.60(\mathrm{dt}, 1 \mathrm{H}, J=13.4$ and $1.9 \mathrm{~Hz}), 2.34-2.30(\mathrm{~m}, 2 \mathrm{H}), 1.99-1.86(\mathrm{~m}$, $2 \mathrm{H}), 1.83(\mathrm{~s}, 3 \mathrm{H}), 1.69-1.47(\mathrm{~m}, 2 \mathrm{H}), 1.44-1.29(\mathrm{~m}, 1 \mathrm{H}), 1.19-1.05(\mathrm{~m}, 1 \mathrm{H}) ;{ }^{13} \mathrm{C} \mathrm{NMR}\left(\mathrm{C}_{6} \mathrm{D}_{6}, 75 \mathrm{MHz}\right) \delta 211.5$, 135.9, 128.7, 127.4, 121.1, 119.6, 119.4, 116.8, 110.8, 50.6, 43.9, 38.1, 35.2, 29.8, 24.4, 11.6; IR (film): 3357, 3053, 2928, 2856, 1695, 1461, 1264, $739 \mathrm{~cm}^{-1}$; HRMS (EI): Exact mass calcd for $\mathrm{C}_{16} \mathrm{H}_{19} \mathrm{NO}[\mathrm{M}]^{+}: 241.1467$. Found: 241.1473. 


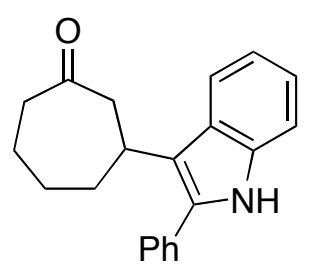

3-(2-Phenyl-1H-indol-3-yl)cycloheptanone (3c)(table 3, entry 3). Isolated $69 \mathrm{mg}$ as a clear, colorless oil after column chromatography $\left(15 \%\right.$ EtOAc/hexanes). TLC $R_{\mathrm{f}} 0.49\left(25 \%\right.$ EtOAc/hexanes); ${ }^{1} \mathrm{H}$ NMR $\left(\mathrm{CDCl}_{3}, 300\right.$ $\mathrm{MHz}) \delta 8.10($ br s, $1 \mathrm{H}), 7.74(\mathrm{~d}, 1 \mathrm{H}, J=7.8 \mathrm{~Hz}), 7.47-7.37(\mathrm{~m}, 6 \mathrm{H}), 7.22($ ap q, $1 \mathrm{H}, J=7.2 \mathrm{~Hz}), 7.14($ ap q, $1 \mathrm{H}, J=7.4 \mathrm{~Hz}), 3.50-3.33(\mathrm{~m}, 2 \mathrm{H}), 2.69(\mathrm{~d}, 1 \mathrm{H}, J=13.2 \mathrm{~Hz}), 2.61-2.56(\mathrm{~m}, 2 \mathrm{H}), 2.34-1.41(\mathrm{~m}, 6 \mathrm{H}) ;{ }^{13} \mathrm{C} \mathrm{NMR}$ $\left(\mathrm{CDCl}_{3}, 75 \mathrm{MHz}\right) \delta 214.6,136.6,133.8,133.5,129.3,129.1,128.4,127.3,122.5,120.9,119.9,118.2,111.6$, 60.0, 44.3, 38.4, 34.9, 30.1, 24.9; IR (film): 3344, 2925, 2853, 1689, 1457, 1275, 764, 749, $699 \mathrm{~cm}^{-1}$; HRMS (EI): Exact mass calcd for $\mathrm{C}_{21} \mathrm{H}_{21} \mathrm{NO}[\mathrm{M}]^{+}:$303.1623. Found: 303.1629.

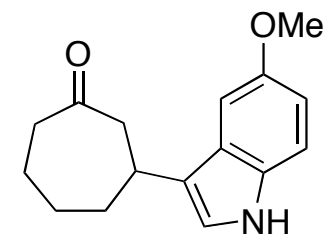

3-(5-Methoxy-1H-indol-3-yl)cycloheptanone (3d)(table 3, entry 4). Isolated $58 \mathrm{mg}$ as a clear, colorless oil after column chromatography (30\% EtOAc/hexanes). TLC $R_{\mathrm{f}} 0.44$ (40\% EtOAc/hexanes); ${ }^{1} \mathrm{H} \mathrm{NMR}\left(\mathrm{CDCl}_{3}\right.$, $300 \mathrm{MHz}) \delta 8.15$ (br s, $1 \mathrm{H}), 7.22(\mathrm{~d}, 1 \mathrm{H}, J=8.8 \mathrm{~Hz}), 7.03(\mathrm{~d}, 1 \mathrm{H}, J=2.3 \mathrm{~Hz}), 6.89(\mathrm{~d}, 1 \mathrm{H}, J=2.3 \mathrm{~Hz}), 6.85$ $(\mathrm{dd}, 1 \mathrm{H}, J=8.8$ and $2.4 \mathrm{~Hz}), 3.86(\mathrm{~s}, 3 \mathrm{H}), 3.19(\mathrm{tt}, 1 \mathrm{H}, J=10.8$ and $2.5 \mathrm{~Hz}), 2.93(\mathrm{t}, 1 \mathrm{H}, J=12.4 \mathrm{H}), 2.83$ (dddd, $1 \mathrm{H}, J=14.4 \mathrm{~Hz}, J=1.6 \mathrm{~Hz}, J=1.5 \mathrm{~Hz}, J=1.4 \mathrm{~Hz}), 2.63(\mathrm{q}, 2 \mathrm{H}, J=4.4 \mathrm{~Hz}), 2.28(\mathrm{~d}, 1 \mathrm{H}, J=14.0 \mathrm{~Hz}$ ), 2.09-1.95 (m, 2H), 1.83-1.51 (m, 3H); ${ }^{13} \mathrm{C} \mathrm{NMR}\left(\mathrm{CDCl}_{3}, 75 \mathrm{MHz}\right) \delta 214.9,154.2,132.0,126.8,121.5,121.0$, 112.5, 112.4, 101.4, 56.4, 50.9, 44.5, 38.4, 34.3, 29.6, 24.8; IR (film): 3366, 2930, 2855, 1691, 1583, 1485, 1441, 1211, 1052, 796, $749 \mathrm{~cm}^{-1}$; HRMS (EI): Exact mass calcd for $\mathrm{C}_{16} \mathrm{H}_{19} \mathrm{NO}_{2}[\mathrm{M}]^{+}$: 257.1416. Found: 257.1406.

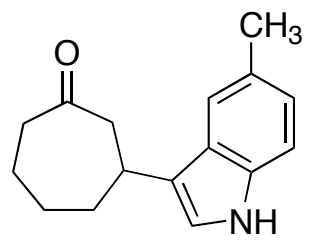

3-(5-Methyl-1H-indol-3-yl)cycloheptanone (3e)(table 3, entry 5). Isolated $53 \mathrm{mg}$ as a clear, colorless oil after column chromatography $\left(\mathrm{CH}_{2} \mathrm{Cl}_{2}\right)$. TLC $R_{\mathrm{f}} 0.33\left(25 \%\right.$ EtOAc/hexanes); ${ }^{1} \mathrm{H}$ NMR $\left(\mathrm{C}_{6} \mathrm{D}_{6}, 300 \mathrm{MHz}\right) \delta 7.51(\mathrm{~d}$, $1 \mathrm{H}, J=0.7 \mathrm{~Hz}), 7.08(\mathrm{dd}, 1 \mathrm{H}, J=8.4$ and $1.3 \mathrm{~Hz}), 7.00(\mathrm{~d}, 1 \mathrm{H}, J=8.3 \mathrm{~Hz}), 6.57(\mathrm{~s}, 1 \mathrm{H}), 6.33(\mathrm{~d}, 1 \mathrm{H}, J=2.1$ $\mathrm{Hz}), 3.08(\mathrm{tt}, 1 \mathrm{H}, J=10.4$ and $2.5 \mathrm{~Hz}), 2.83(\mathrm{ddd}, 1 \mathrm{H}, J=14.4,3.3$, and $1.6 \mathrm{~Hz}), 2.74(\mathrm{dd}, 1 \mathrm{H}, J=14.4 \mathrm{and}$ $10.9 \mathrm{~Hz}), 2.44(\mathrm{~s}, 3 \mathrm{H}), 2.31(\mathrm{dd}, 2 \mathrm{H}, J=8.5$ and $4.6 \mathrm{~Hz}), 2.11-2.03(\mathrm{~m}, 1 \mathrm{H}), 1.67-1.43(\mathrm{~m}, 3 \mathrm{H}), 1.37-1.11(\mathrm{~m}$, $2 \mathrm{H}) ;{ }^{13} \mathrm{C}$ NMR $\left(\mathrm{C}_{6} \mathrm{D}_{6}, 75 \mathrm{MHz}\right) \delta 211.5,135.5,128.1,127.2,124.2,121.4,119.9,119.4,111.4,50.8,44.1,38.6$, 34.4, 29.5, 24.9, 22.0; IR (film): 3367, 2926, 2856, 1698, 1444, 1347, $795 \mathrm{~cm}^{-1}$; HRMS (EI): Exact mass calcd for $\mathrm{C}_{16} \mathrm{H}_{19} \mathrm{NO}[\mathrm{M}]^{+}:$241.1467. Found: 241.1460. 


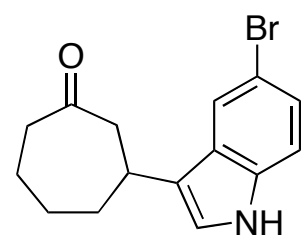

3-(5-Bromo-1H-indol-3-yl)cycloheptanone (3f)(table 3, entry 6). Isolated $60 \mathrm{mg}$ as a clear, colorless oil after column chromatography $\left(\mathrm{CH}_{2} \mathrm{Cl}_{2}\right)$. TLC $R_{\mathrm{f}} 0.31$ (30\% EtOAc:hexanes); ${ }^{1} \mathrm{H}$ NMR $\left(\mathrm{C}_{6} \mathrm{D}_{6}, 300 \mathrm{MHz}\right) \delta 7.86(\mathrm{~d}$, $1 \mathrm{H}, J=1.9 \mathrm{~Hz}), 7.33(\mathrm{dd}, 1 \mathrm{H}, J=8.7$ and $1.8 \mathrm{~Hz}), 6.71(\mathrm{dd}, 1 \mathrm{H}, J=8.6$ and $0.4 \mathrm{~Hz}), 6.67(\mathrm{br} \mathrm{s}, 1 \mathrm{H}), 6.23(\mathrm{br} \mathrm{d}$, $1 \mathrm{H}, J=2.3 \mathrm{~Hz}), 2.87-2.78(\mathrm{~m}, 1 \mathrm{H}), 2.62-2.60(\mathrm{~m}, 2 \mathrm{H}), 2.34-2.17(\mathrm{~m}, 2 \mathrm{H}), 1.89-1.83(\mathrm{~m}, 1 \mathrm{H}), 1.53-1.02(\mathrm{~m}$, $5 \mathrm{H}) ;{ }^{13} \mathrm{C}$ NMR $\left(\mathrm{C}_{6} \mathrm{D}_{6}, 75 \mathrm{MHz}\right) \delta 211.2,135.3,125.2,122.1,121.4,121.0,113.01,112.99,50.2,43.8,38.4$, 33.7, 29.1, 24.4; IR (film): 3348, 2926, 2855, 1692, 1459, 1275, 1260, 884, $749 \mathrm{~cm}^{-1}$; HRMS (EI): Exact mass calcd for $\mathrm{C}_{15} \mathrm{H}_{16} \mathrm{BrNO}[\mathrm{M}]^{+}: 305.0415$. Found: 305.0399 .

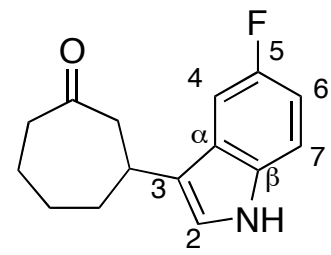

3-(5-Fluoro-1H-indol-3-yl)cycloheptanone (3g)(table 3, entry 7). Isolated $36 \mathrm{mg}$ as a clear, colorless oil after column chromatography $(30 \%$ EtOAc/hexanes $)$ followed by column chromatography with $1 \%$ $\mathrm{MeOH} /$ dichloromethane. TLC $R_{\mathrm{f}} 0.31$ (30\% EtOAc:hexanes); ${ }^{1} \mathrm{H}$ NMR $\left(\mathrm{C}_{6} \mathrm{D}_{6}, 300 \mathrm{MHz}\right) \delta 7.33(\mathrm{dd}, 1 \mathrm{H}, J=$ 9.7 and $2.3 \mathrm{~Hz}), 6.97(\mathrm{td}, 1 \mathrm{H}, J=8.9$ and $2.4 \mathrm{~Hz}), 6.80($ ap dd, $2 \mathrm{H}, J=8.8$ and $4.4 \mathrm{~Hz}), 6.33(\mathrm{~d}, 1 \mathrm{H}, J=2.2 \mathrm{~Hz})$, 2.91-2.83 (m, 1H), 2.65 (ap d, 2H, $J=7.6 \mathrm{~Hz}), 2.30-2.24(\mathrm{~m}, 2 \mathrm{H}), 1.91$ (ap d, 1H, $J=14.9 \mathrm{~Hz}), 1.56-1.29(\mathrm{~m}$, $5 \mathrm{H}) ;{ }^{13} \mathrm{C}$ NMR $\left(\mathrm{C}_{6} \mathrm{D}_{6}, 75 \mathrm{MHz}\right) \delta 211.5,158.2(\mathrm{~d}, J=234.1 \mathrm{~Hz}, \mathrm{C}(5)-\mathrm{F}), 133.3,126.8(\mathrm{~d}, J=9.4 \mathrm{~Hz}, \mathrm{C}(\alpha)-\mathrm{F})$, 121.7 (d, $J=4.8 \mathrm{~Hz}, \mathrm{C}(3)-\mathrm{F}), 121.6,112.2$ (d, $J=9.6 \mathrm{~Hz}, \mathrm{C}(7)-\mathrm{F}), 110.6$ (d, $J=26.2 \mathrm{~Hz}, \mathrm{C}(6)-\mathrm{F}), 104.5$ (d, $J=$ $23.3 \mathrm{~Hz}, \mathrm{C}(4)-\mathrm{F}), 50.2,43.8,38.2,33.9,29.1,24.4$; IR (film): 3349, 2928, 2856, 1689, 1581, 1486, 1456, 1347, 1259, 1161, 1100, 938, 850, 796, 763, $749 \mathrm{~cm}^{-1}$; HRMS (EI): Exact mass calcd for $\mathrm{C}_{15} \mathrm{H}_{16} \mathrm{FNO}[\mathrm{M}]^{+}: 245.1216$. Found: 245.1201 .

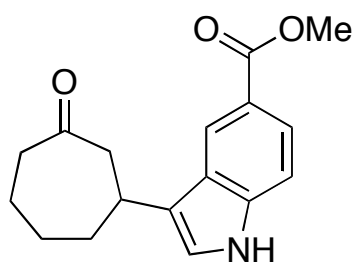

Methyl 3-(3-oxocycloheptyl)-1H-indole-5-carboxylate (3h)(table 3, entry 8). Isolated $28 \mathrm{mg}$ as a clear, colorless oil after column chromatography (40\% EtOAc/hexanes). TLC $R_{\mathrm{f}} 0.20$ (30\% EtOAc/hexanes); ${ }^{1} \mathrm{H}$ NMR $\left(\mathrm{CDCl}_{3}, 300 \mathrm{MHz}\right) \delta 8.34(\mathrm{br} \mathrm{s}, 2 \mathrm{H}), 7.88(\mathrm{~d}, 1 \mathrm{H}, J=8.4 \mathrm{~Hz}), 7.34(\mathrm{~d}, 1 \mathrm{H}, J=8.6 \mathrm{~Hz}), 7.00$ (s, $\left.1 \mathrm{H}\right), 3.92$ $(\mathrm{s}, 3 \mathrm{H}), 3.30$ (br ap t, $1 \mathrm{H}, J=13.1 \mathrm{~Hz}), 2.95(\mathrm{dd}, 1 \mathrm{H}, J=14.7$ and $11.4 \mathrm{~Hz}), 2.82(\mathrm{~d}, 1 \mathrm{H}, J=14.5 \mathrm{~Hz}), 2.63($ ap $\mathrm{dt}, 2 \mathrm{H}, J=7.8$ and $3.8 \mathrm{~Hz}), 2.26(\mathrm{br} \mathrm{d}, 1 \mathrm{H}, J=13.4 \mathrm{~Hz}), 2.08-1.95(\mathrm{~m}, 2 \mathrm{H}), 1.85-1.58(\mathrm{~m}, 3 \mathrm{H})$; ${ }^{13} \mathrm{C} \mathrm{NMR}$ $\left(\mathrm{C}_{6} \mathrm{D}_{6}, 75 \mathrm{MHz}\right) \delta 211.5,168.0,139.4,126.3,123.9,123.1,122.5,122.1,121.3,111.4,51.5,50.4,43.8,38.6$, 33.8, 29.1, 24.4; IR (film): 3339, 2926, 2855, 1697, 1437, 1275, 1260, 1109, 764, $750 \mathrm{~cm}^{-1}$; HRMS (EI): Exact mass calcd for $\mathrm{C}_{17} \mathrm{H}_{19} \mathrm{NO}_{3}[\mathrm{M}]^{+}: 285.1365$. Found: 285.1369 . 
Table 4a. Experimental Details for Results in Table 4

\begin{tabular}{cccccc}
\hline Entry & $\mathrm{m}_{\text {enone }}(\mathrm{g})$ & $\mathrm{n}_{\text {enone }}(\mathrm{mmol})$ & $\mathrm{m}_{\text {indole }}(\mathrm{g})$ & $\mathrm{n}_{\text {indole }}(\mathrm{mmol})$ & $\mathrm{V}_{\text {solvent }}(\mathrm{mL})$ \\
\hline 1 & 0.105 & 0.949 & 0.117 & 0.999 & 19 \\
2 & 0.050 & 0.45 & 0.060 & 0.45 & 9.0 \\
3 & 0.050 & 0.45 & 0.071 & 0.45 & 9.0 \\
4 & 0.050 & 0.45 & 0.094 & 0.45 & 9.0 \\
5 & 0.050 & 0.45 & 0.087 & 0.55 & 9.0 \\
6 & 0.050 & 0.45 & 0.118 & 0.55 & 9.0 \\
\hline
\end{tabular}

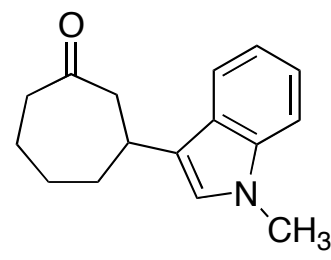

3-(1-Methyl-3-indolyl)-cycloheptanone (3k)(table 4, entry 2). Isolated $56 \mathrm{mg}$ as a clear, colorless oil after

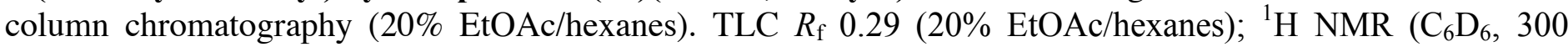
$\mathrm{MHz}) \delta 7.67(\mathrm{~d}, 1 \mathrm{H}, J=7.9 \mathrm{~Hz}), 7.29-7.17(\mathrm{~m}, 2 \mathrm{H}), 7.03(\mathrm{~d}, 1 \mathrm{H}, J=7.6 \mathrm{~Hz}), 6.27(\mathrm{~s}, 1 \mathrm{H}), 3.10(\operatorname{apt} \mathrm{t}, 1 \mathrm{H}, J=$ 10.7 and $2.9 \mathrm{~Hz}), 2.94(\mathrm{~s}, 3 \mathrm{H}), 2.82-2.72(\mathrm{~m}, 2 \mathrm{H}), 2.33(\mathrm{dd}, 2 \mathrm{H}, J=9.7$ and $4.7 \mathrm{~Hz}), 2.11-2.05(\mathrm{~m}, 1 \mathrm{H}), 1.68-$ 1.47 (m, 3H), 1.40-1.14 (m, 2H); ${ }^{13} \mathrm{C}$ NMR $\left(\mathrm{C}_{6} \mathrm{D}_{6}, 75 \mathrm{MHz}\right) \delta 211.4,137.9,127.4,124.5,122.2,120.7,119.9$, 119.4, 109.8, 51.0, 44.1, 38.8, 34.3, 32.0, 29.5, 24.8; IR (film): 3052, 2927, 2855, 1697, 1472, 1326, 1262, 1154, $740 \mathrm{~cm}^{-1}$; HRMS (EI): Exact mass calcd for $\mathrm{C}_{16} \mathrm{H}_{19} \mathrm{NO}[\mathrm{M}]^{+}: 241.1467$. Found: 241.1479.

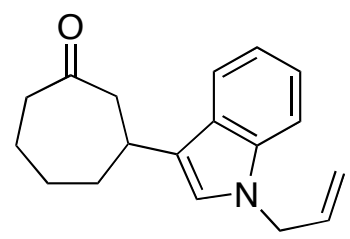

3-(1-Allyl-3-indolyl)-cycloheptanone (3I)(table 4, entry 3). Isolated $64 \mathrm{mg}$ as a clear, colorless oil after

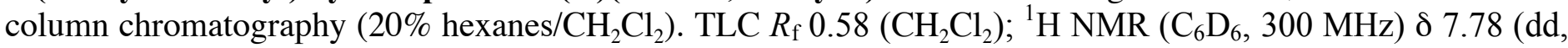
$1 \mathrm{H}, J=6.8$ and $1.1 \mathrm{~Hz}), 7.38-7.29(\mathrm{~m}, 4 \mathrm{H}), 6.56(\mathrm{~s}, 1 \mathrm{H}), 5.65(\mathrm{ddt}, 1 \mathrm{H}, J=17.0,10.4$ and $5.3 \mathrm{~Hz}), 4.95$ (ddd, $1 \mathrm{H}, J=10.2,2.9$ and $1.4 \mathrm{~Hz}), 4.85(\mathrm{ddd}, 1 \mathrm{H}, J=17.1,3.1$ and $1.6 \mathrm{~Hz}), 4.16(\mathrm{dt}, 2 \mathrm{H}, J=5.3$ and $1.6 \mathrm{~Hz}), 3.21$ (tt, $1 \mathrm{H}, J=10.6,2.7 \mathrm{~Hz}), 2.94(\mathrm{ddd}, 1 \mathrm{H}, J=14.4,3.3,1.6 \mathrm{~Hz}), 2.86(\mathrm{dd}, 1 \mathrm{H}, J=14.4$ and $10.9 \mathrm{~Hz}), 2.44(\mathrm{dd}$, $1 \mathrm{H}, J=9.3$ and $4.4 \mathrm{~Hz}), 2.22-2.16(\mathrm{~m}, 1 \mathrm{H}), 1.79-1.24(\mathrm{~m}, 5 \mathrm{H}) ;{ }^{13} \mathrm{C} \mathrm{NMR}\left(\mathrm{C}_{6} \mathrm{D}_{6}, 75 \mathrm{MHz}\right) \delta 211.2,137.2$, 134.0, 127.3, 123.4, 122.1, 121.0, 119.8, 119.4, 116.6, 110.1, 50.7, 48.4, 43.9, 38.5, 34.5, 29.4, 24.5; IR (film): 3052, 2926, 2856, 1697, 1644, 1613, 1466, 1331, 1266, 992, $740 \mathrm{~cm}^{-1}$; HRMS (EI): Exact mass calcd for $\mathrm{C}_{18} \mathrm{H}_{21} \mathrm{NO}[\mathrm{M}]^{+}:$267.1623. Found: 267.1601. 


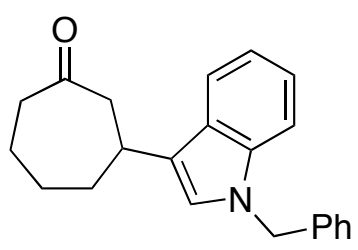

3-(1-Benzyl-3-indolyl)-cycloheptanone (3m)(table 4, entry 4). Isolated $47 \mathrm{mg}$ as a clear, colorless oil after column chromatography $\left(30 \%\right.$ hexanes $\left./ \mathrm{CH}_{2} \mathrm{Cl}_{2}\right)$. TLC $R_{\mathrm{f}} 0.58\left(\mathrm{CH}_{2} \mathrm{Cl}_{2}\right) ;{ }^{1} \mathrm{H}$ NMR $\left(\mathrm{C}_{6} \mathrm{D}_{6}, 300 \mathrm{MHz}\right) \delta 7.81-7.77$ $(\mathrm{m}, 1 \mathrm{H}), 7.31-7.29(\mathrm{~m}, 4 \mathrm{H}), 7.21-7.16(\mathrm{~m}, 1 \mathrm{H}), 7.12-7.08(\mathrm{~m}, 3 \mathrm{H}), 6.93-6.90(\mathrm{~m}, 2 \mathrm{H}), 6.57(\mathrm{~s}, 1 \mathrm{H}), 4.79(\mathrm{~s}, 2 \mathrm{H})$, 3.21 (ap tt, $1 \mathrm{H}, J=13.7$ and $2.7 \mathrm{~Hz}), 2.97-2.91(\mathrm{~m}, 1 \mathrm{H}), 2.83(\mathrm{dd}, 1 \mathrm{H}, J=14.3$ and $11.1 \mathrm{~Hz}), 2.43(\mathrm{ap} \mathrm{dd}, 2 \mathrm{H}, J$ $=9.8$ and $4.7 \mathrm{~Hz}), 2.18(\mathrm{br} \mathrm{d}, 1 \mathrm{H}, J=13.9 \mathrm{~Hz}), 1.77-1.54(\mathrm{~m}, 3 \mathrm{H}), 1.49-1.22(\mathrm{~m}, 2 \mathrm{H}) ;{ }^{13} \mathrm{C} \mathrm{NMR}\left(\mathrm{C}_{6} \mathrm{D}_{6}, 75\right.$ $\mathrm{MHz}) \delta 211.0,138.1,137.3,128.7,127.3,126.7,123.7,122.2,121.2,119.7,119.5,110.0,50.6,49.5,43.8$, 38.3, 34.1, 29.3, 24.4; IR (film): 3055, 3029, 2926, 2856, 1696, 1466, 1453, 1347, 1331, 1264, $1178,1015,741$ $703 \mathrm{~cm}^{-1}$; HRMS (EI): Exact mass calcd for $\mathrm{C}_{22} \mathrm{H}_{23} \mathrm{NO}[\mathrm{M}]^{+}: 317.1780$. Found: 317.1783 .

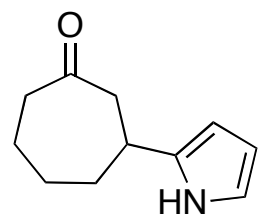

3-(1H-Pyrrol-2-yl)cycloheptanone (13)(eq 4). A borosilicate tube was charged with a stir bar, cyclohept-2enone $(0.100 \mathrm{~g}, 0.907 \mathrm{mmol})$ and pyrrole $(0.182 \mathrm{~g}, 2.72 \mathrm{mmol})$. A volume of $6.0 \mathrm{~mL}$ dichloromethane was added to the mixture and the volume was marked on the tube. An excess of 1-2 mL of solvent was then added to the tube. The tube was capped with a septum and was purged with a nitrogen balloon and an outlet to the volume marked. The reaction mixture was then placed on a stir-plate and allowed to stir between two exposure panels each equipped with four $8 \mathrm{~W}$ UV-A bulbs for 48 hours to obtain the crude mixture as a dark red liquid. The crude mixture was concentrated under reduced pressure to a few milliliters and was purified by flash chromatography using $25 \%$ ethyl acetate in hexanes. The purified product was a clear, colorless oil $(0.011 \mathrm{~g}$, 7\% yield). TLC $R_{\mathrm{f}} 0.39$ (30\% EtOAc/hexanes); ${ }^{1} \mathrm{H}$ NMR $\left(\mathrm{CDCl}_{3}, 300 \mathrm{MHz}\right) \delta 8.06$ (br s, $\left.1 \mathrm{H}\right), 6.68(\mathrm{dd}, 1 \mathrm{H}, J$ $=3.8$ and $2.3 \mathrm{~Hz}), 6.10(\mathrm{dd}, 1 \mathrm{H}, J=5.8$ and $2.9 \mathrm{~Hz}), 5.92(\mathrm{~s}, 1 \mathrm{H}), 3.00($ ap t, $1 \mathrm{H}, J=10.4$ and $3.3 \mathrm{~Hz}), 2.89$ $2.73(\mathrm{~m}, 2 \mathrm{H}), 2.53(\mathrm{~d}, 1 \mathrm{H}, J=4.9 \mathrm{~Hz}), 2.50(\mathrm{~d}, 1 \mathrm{H}, J=4.4 \mathrm{~Hz}), 2.17($ ap br d, $1 \mathrm{H}, J=13.3 \mathrm{~Hz}), 2.03-1.87(\mathrm{~m}$, $2 \mathrm{H}), 1.82-1.44(\mathrm{~m}, 3 \mathrm{H}) ;{ }^{13} \mathrm{C} \mathrm{NMR}\left(\mathrm{CDCl}_{3}, 75 \mathrm{MHz}\right) \delta$ 214.0, 117.1, 108.6, 104.3, 53.83, 50.18, 44.4, 37.6, 35.6, 28.6, 24.4; IR (film): 3374, 2928, 2856, 1692, 1275, 764, 750, $718 \mathrm{~cm}^{-1}$; HRMS (EI): Exact mass calcd for $\mathrm{C}_{11} \mathrm{H}_{15} \mathrm{NO}: 177.1154$. Found: 177.1156 .

Procedure for Attempted Low Temperature Generation/Trapping of (E)-cyclooct-2-enone. ${ }^{13,14}$ A borosilicate tube was charged with a stir bar and $(Z)$-cyclooct-2-enone $(0.033 \mathrm{~g}, 0.27 \mathrm{mmol})$. A volume of 5.4 $\mathrm{mL}$ dichloromethane was added and the volume was marked on the tube. An excess of 1-2 $\mathrm{mL}$ of solvent was then added to the tube. The tube was capped with a septum and was purged with stirring using a nitrogen

(13) For examples of low temperature generation/trapping experiments with (E)-cycloalkenones, see: (a) Corey, E. J.; Tada, M.; LaMahieu, R.; Libit, L. J. Am. Chem. Soc. 1965, 87, 2051. (b) Noyori, R.; Watanabe, A.; Katô, M. Tetrahedron Lett. 1968, 52, 5443. (c) Katô, M.; Noyori, R. Bull. Chem. Soc. Jpn. 1974, 47, 1460.

(14) For examples of low temperature generation/trapping experiments with (E)-cycloalkenes, see: (a) Inoue, Y.; Ueoka, T.; Kuroda, T.; Hakushi, T. J. Chem. Soc., Chem. Commun. 1981, 1031. (b) Inoue, Y.; Ueoka, T.; Kuroda, T.; Hakushi, T. J. Chem. Soc., Perkin Trans. 2 1983, 983. (c) Steinmetz, M. G.; Seguin, K. J.; Udayakumar, B. S.; Behnke, J. S. J. Am. Chem. Soc. 1990, 112, 6601. 
balloon and an outlet until the solvent evaporated to the volume marked. The solution was cooled to $-75^{\circ} \mathrm{C}$ and was then placed on a stir-plate and allowed to stir between two exposure panels each equipped with four $8 \mathrm{~W}$ UV-A bulbs for 20 minutes, maintaining cooling. ${ }^{15}$ Irradiation was then stopped, and a heterogeneous mixture of indole $(0.034 \mathrm{~g}, 0.242 \mathrm{mmol})$ in $1.0 \mathrm{~mL}$ dichloromethane, pre-cooled to $-75^{\circ} \mathrm{C}$, was then added to the borosilicate tube. The reaction mixture was then allowed to gradually warm to room temperature while stirring for 45 minutes. No reaction was observed by TLC. The solvent was evaporated under reduced pressure. ${ }^{1} \mathrm{H}$ NMR of the crude reaction mixture indicated only a mixture of starting materials.

Procedure for Oxygen-Induced Quenching of Enone Triplet. A borosilicate tube was charged with a stir bar, cyclohept-2-enone $(0.025 \mathrm{~g}, 0.23 \mathrm{mmol})$ and indole $(0.029 \mathrm{~g}, 0.25 \mathrm{mmol})$. A volume of $4.5 \mathrm{~mL}$ dichloromethane was added to the tube and stirred open to the atmosphere for 1 minute. The tube was capped with a septum and was then placed on a stir-plate and allowed to stir between two exposure panels each equipped with four $8 \mathrm{~W}$ UV-A bulbs for 18 hours. No indole adduct was observed by TLC. The solvent was evaporated under reduced pressure. ${ }^{1} \mathrm{H}$ NMR of the crude reaction mixture indicated a mixture of starting materials as well as a modest amount of dimers of cyclohept-2-enone. ${ }^{13 a}$

\section{Discussion of Mechanistic Alternatives}

Scheme 2. Proposed photoinduced electron transfer mechanism for the 1,4-addition of indoles to enones

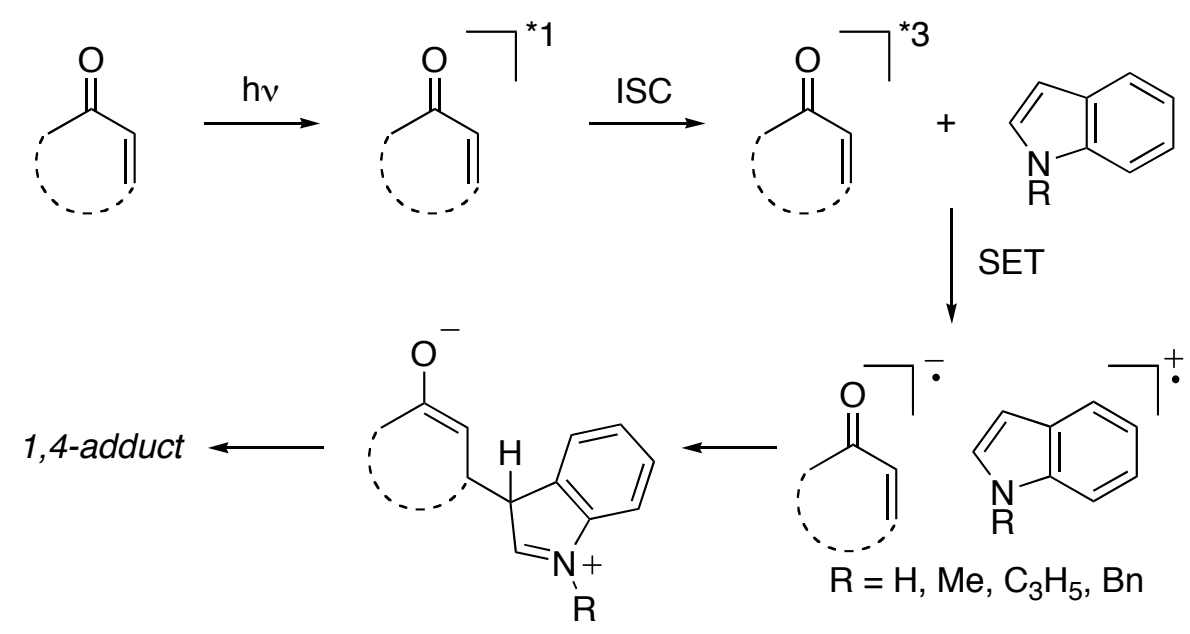

We favor the PET mechanism presented in Scheme 2 over a [2+2] cycloaddition/cyclobutane ring opening sequence that could afford the same products. Such a mechanistic scenario is not consistent with the observed electronic effects on the indole moiety. In addition, the regiochemistry observed in enone [2+2] cycloadditions usually favors formation of the cycloadduct in which the carbonyl and the electron rich group are in a head to

(15) The sample was immersed in a $95 \%$ ethanol bath contained in a $5 \mathrm{~L}$ quartz Dewar and cooled to $-75^{\circ} \mathrm{C}$ by an FTS Systems cold finger apparatus. 
tail configuration, which could not form the formal 1,4-adduct upon cyclobutane ring opening. During the course of these studies, we have not observed formation of [2+2] cycloadducts between indoles and enones. ${ }^{16}$

In specific cases, other mechanisms could also be operating. For example, cyclohept-2-enone and cyclooct-2enone could also afford the observed 1,4-addition product through an $E$-cycloalkenone intermediate. ${ }^{13}$ However, low temperature generation/trapping experiments have been performed with E-cyclooct-2-enone and have resulted in recovery of the starting materials, ${ }^{13}$ suggesting $E$-cycloalkenones are not likely intermediates for these substrates. We also speculate that reaction of methyl vinyl ketone (MVK) via a Bauld-Steckhan type cycloaddition mechanism, ${ }^{17}$ namely a formal hetero-Diels-Alder reaction of MVK with the indole radical cation (affording the 1,4-adduct upon hydrolysis), is unlikely and not consistent with the substrate scope of the reaction.

(16) For examples of [2+2] cycloadditions between $N$-acylindoles and alkenes, see: Weedon, A. C.; Zhang, B. Synthesis 1992, 95 and references cited therein.

(17) (a) Bauld, N. L; Bellville, D. J.; Harirchian, B.; Lorenz, K. T.; Pabon, R. A. Jr.; Reynolds, D. W.; Wirth, D. D.; Chiou, H.-S.; Marsh, B. K. Acc. Chem. Res. 1987, 20, 371. (b) Mlcoch, J.; Steckhan, E. Angew. Chem., Int. Ed. Engl. 1985, 24, 412. 
<smiles>O=C1CCCCC(c2c[nH]c3ccccc23)C1</smiles>

Table 2, Entry 4

$3 a$

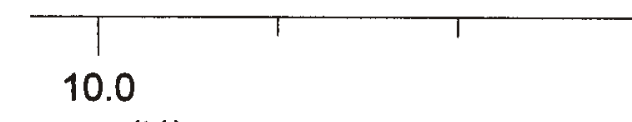

ppm (t1)

$\stackrel{n}{\check{n}}$

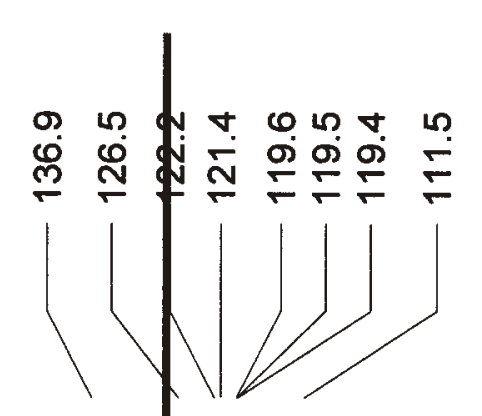

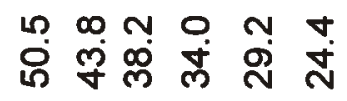
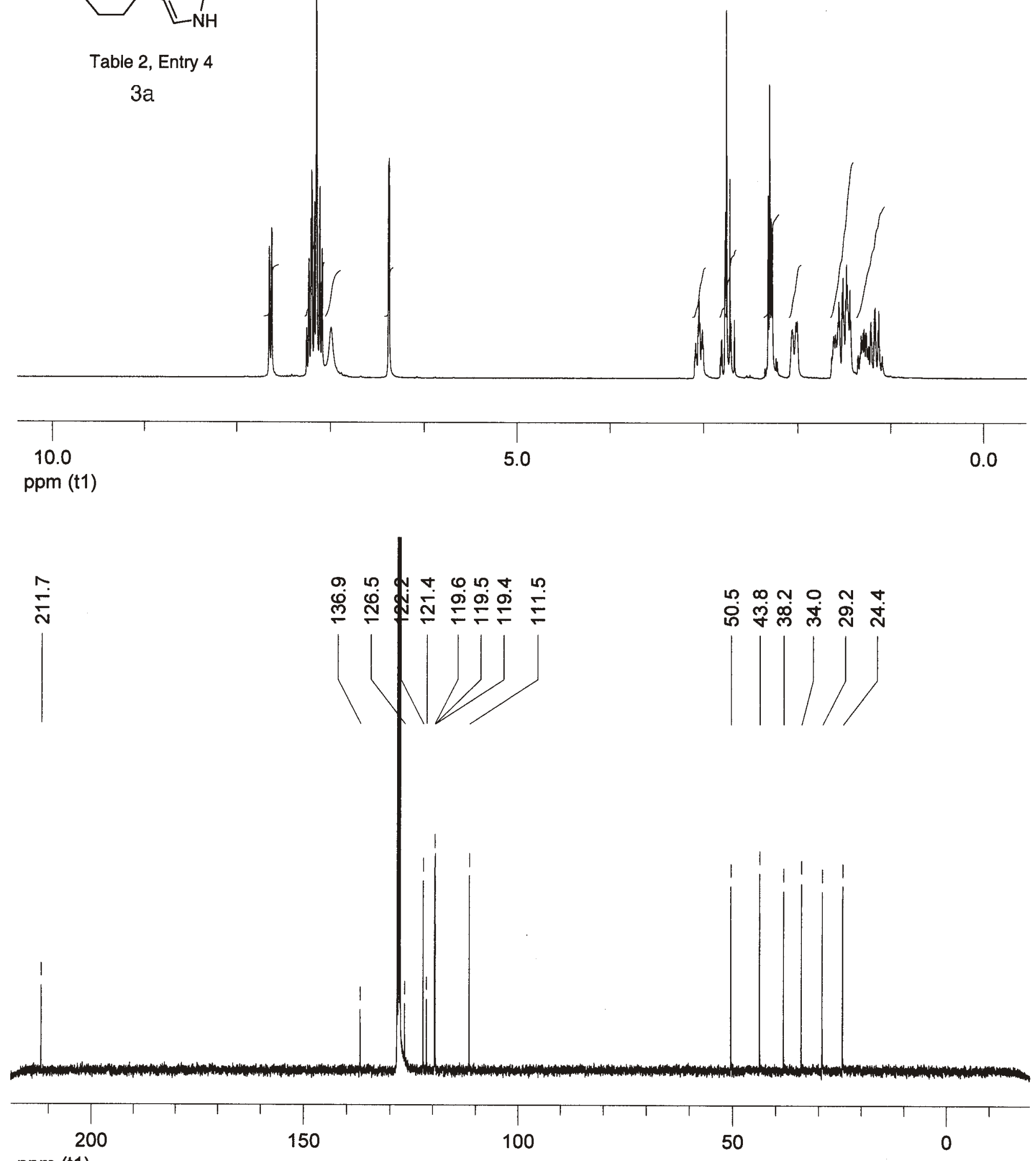

ppm (t1) 


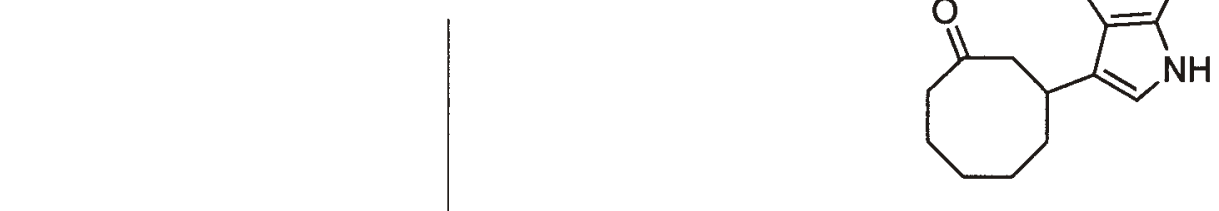

Table 2, Entry 5

11
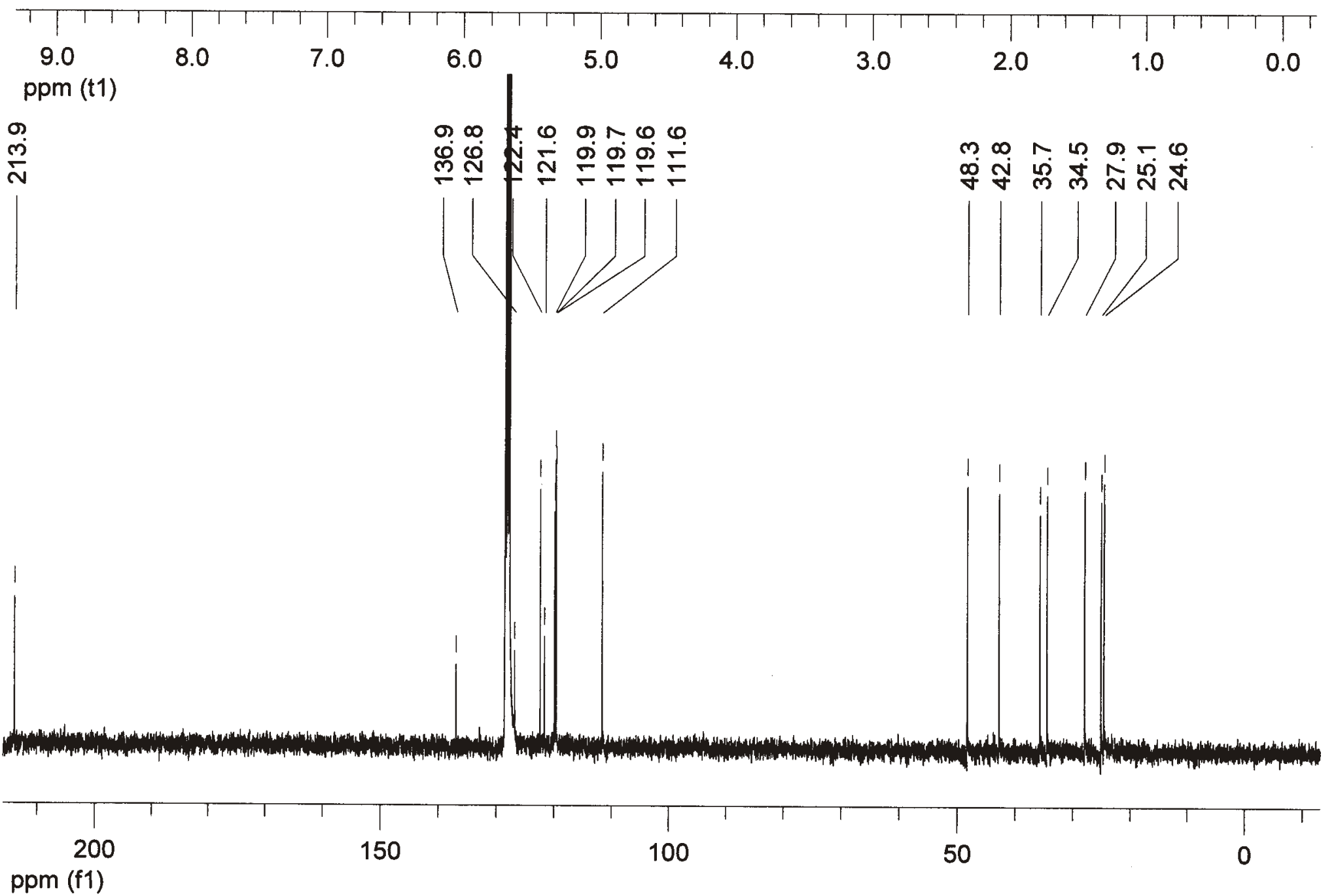


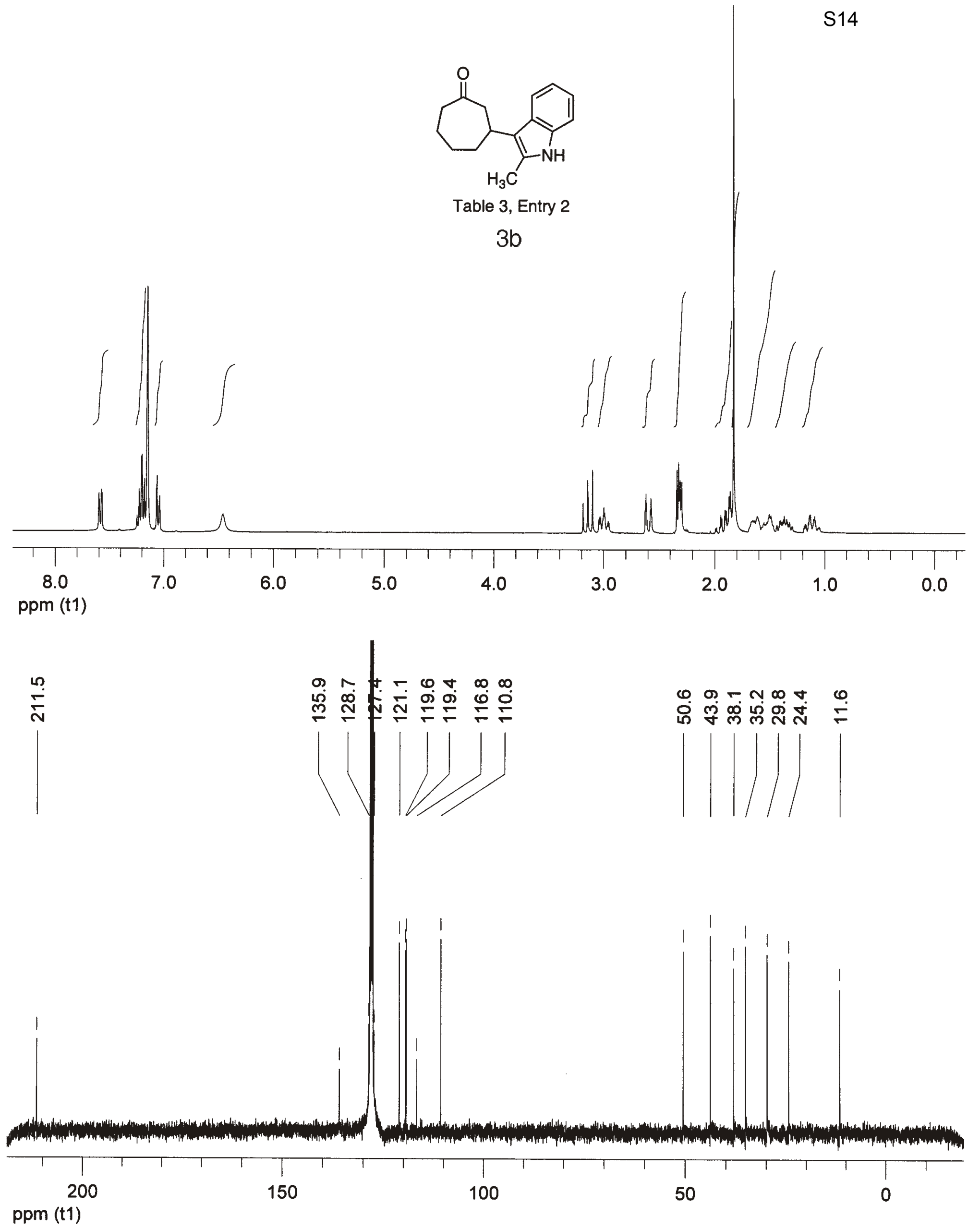




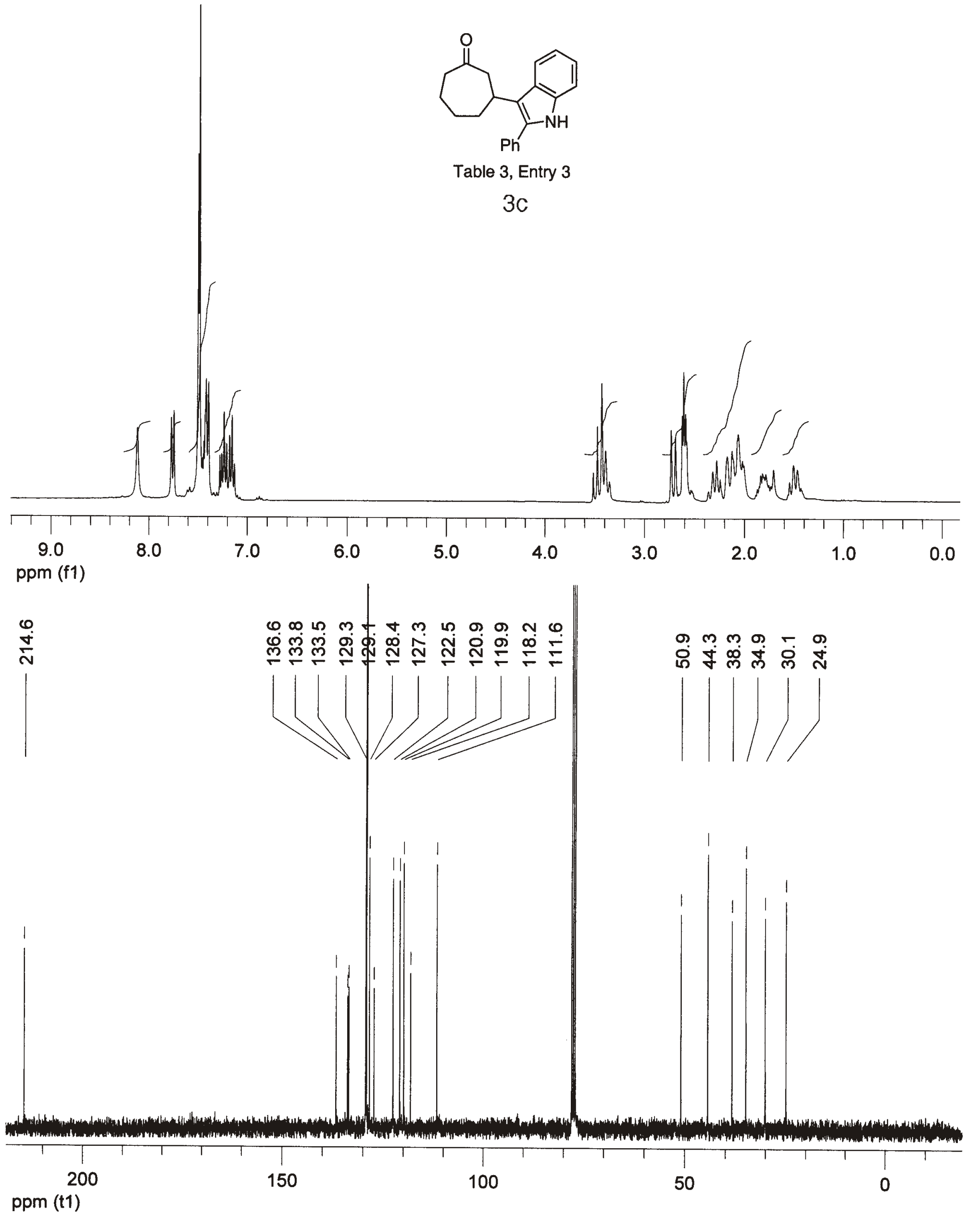


<smiles>COc1ccc2[nH]cc(C3CCCCC(=O)C3)c2c1</smiles>

Table 3, Entry 4

$3 d$
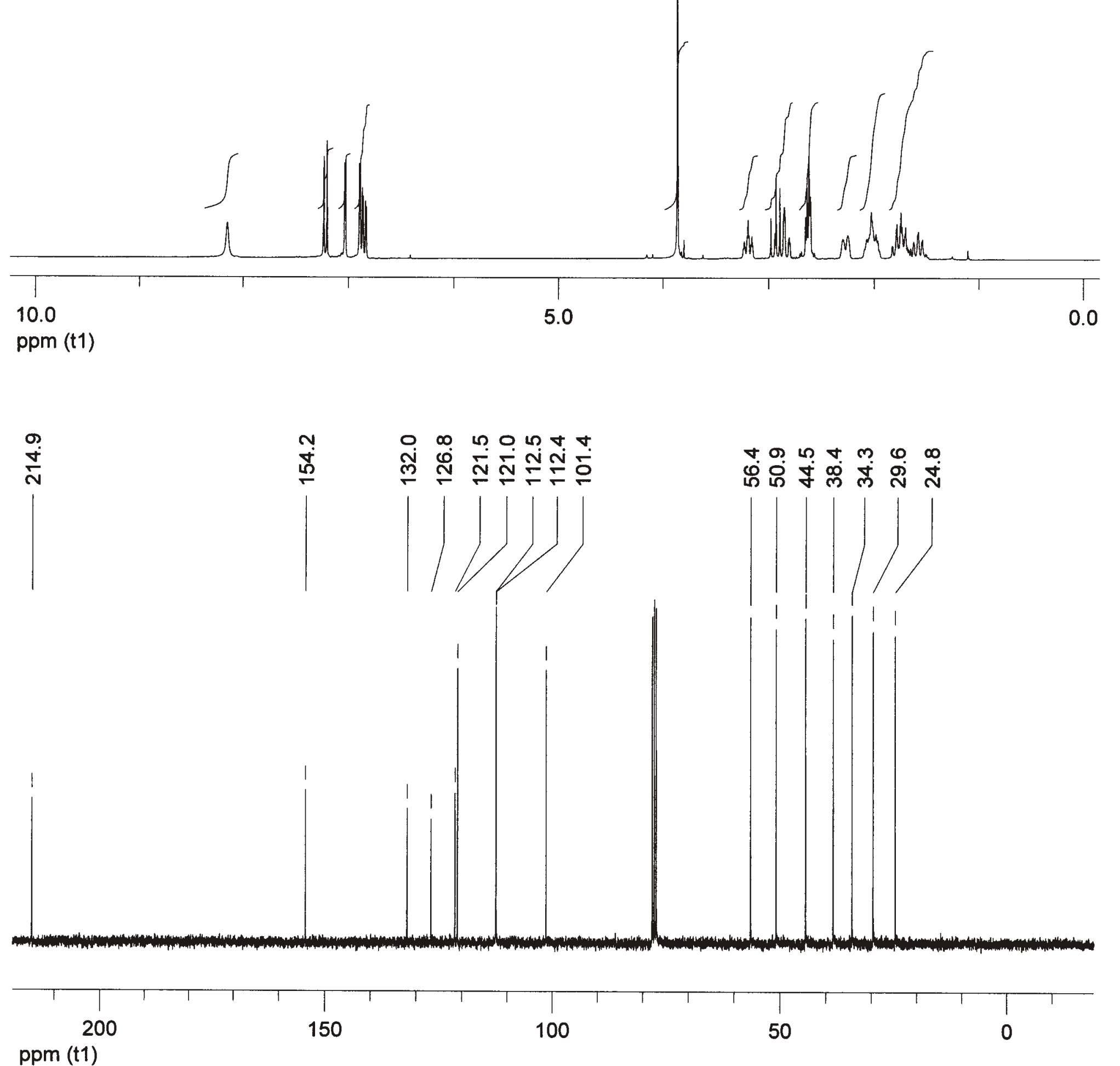


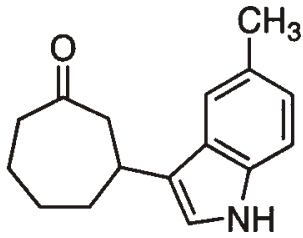

Table 3, Entry 5 $3 \mathrm{e}$

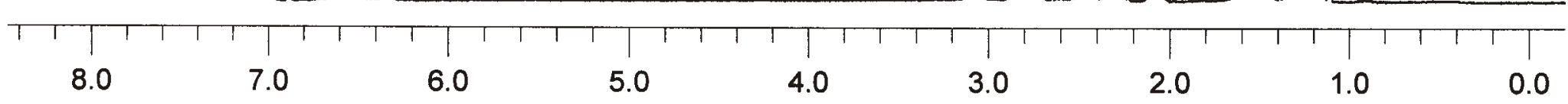

ppm (t1)

$\frac{1}{\pi}$

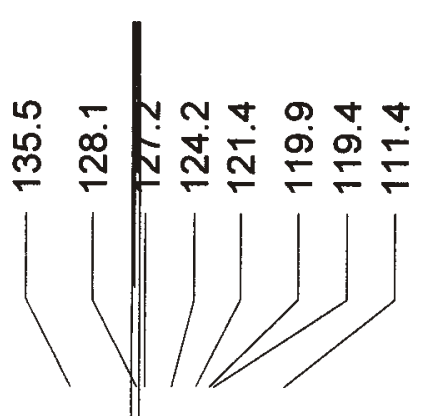

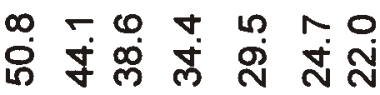
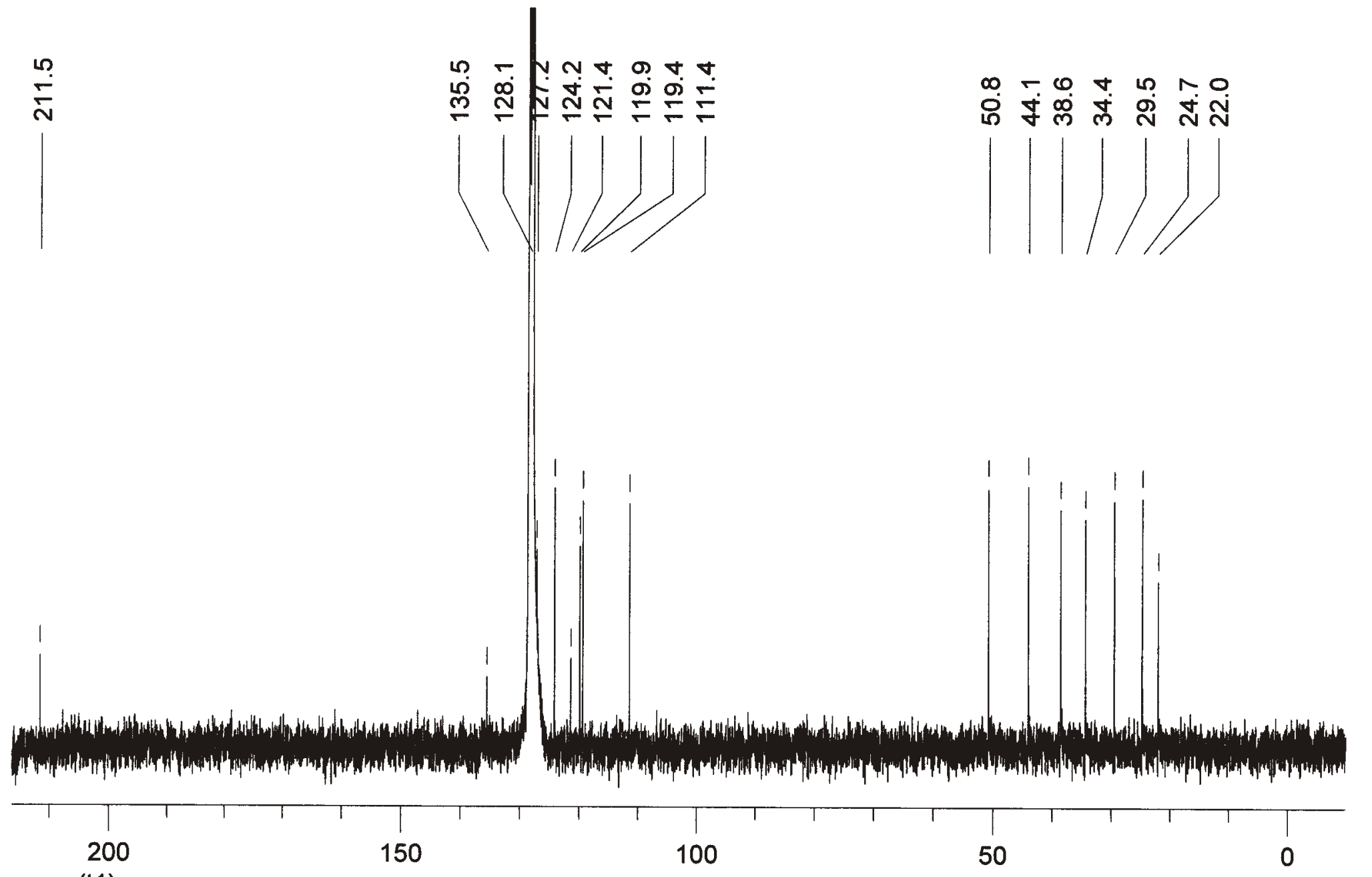

ppm (t1) 


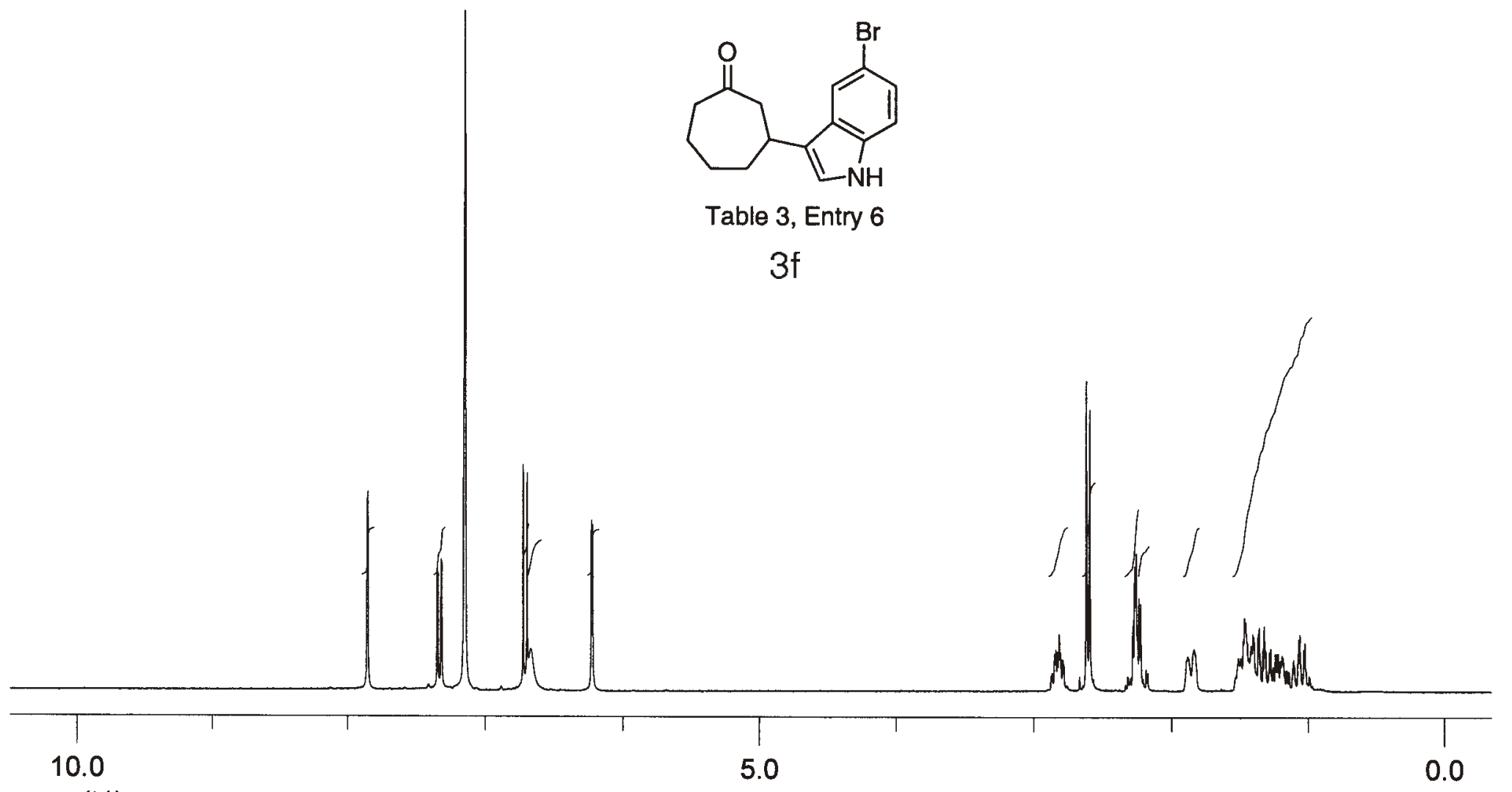

ppm (t1)
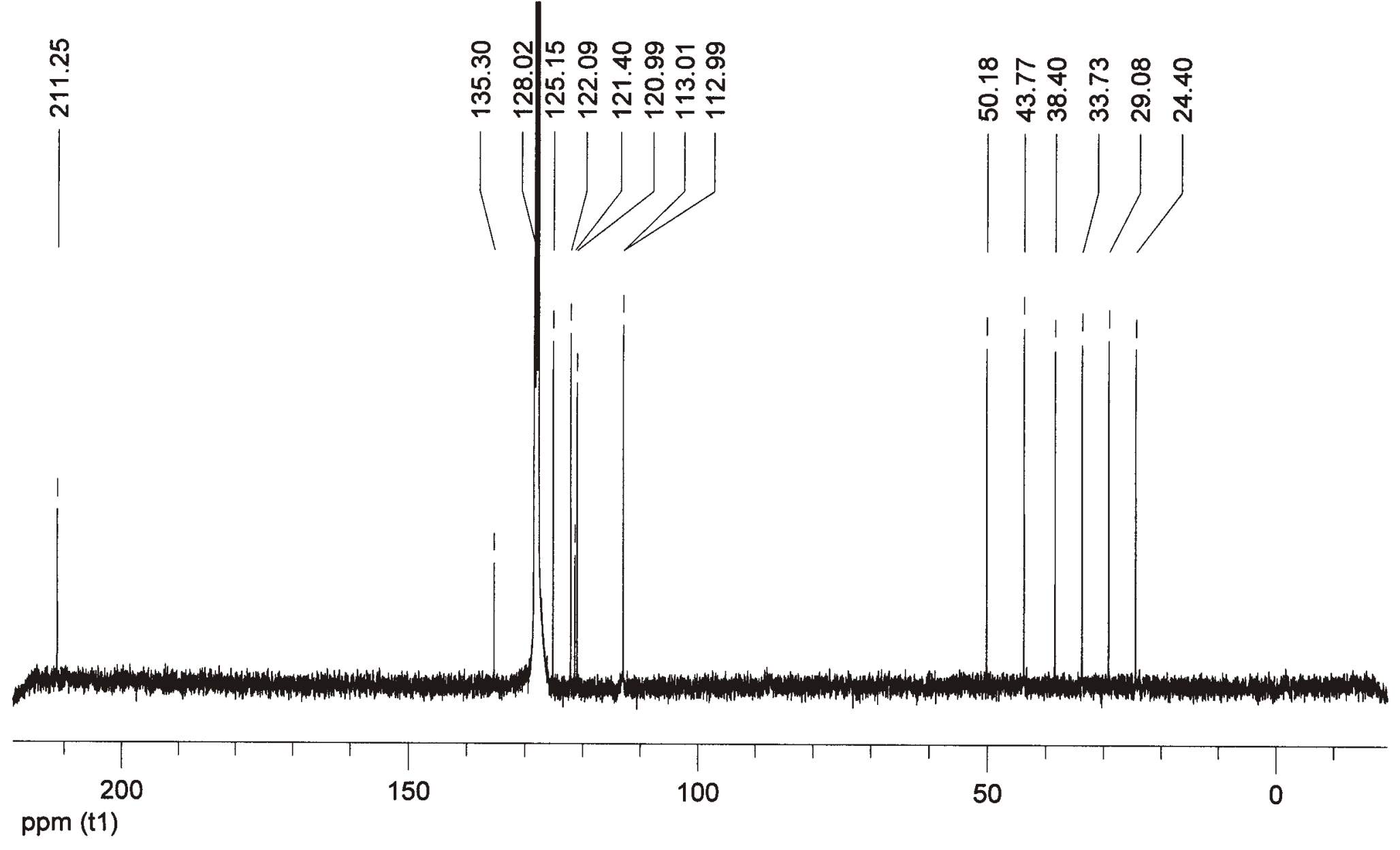
<smiles>O=C1CCCCC(c2c[nH]c3cc(F)ccc23)C1</smiles>

Table 3, Entry 7

$3 \mathrm{~g}$

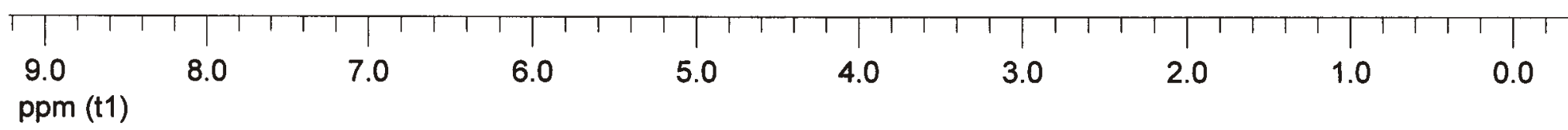

\section{ppm (t1)}

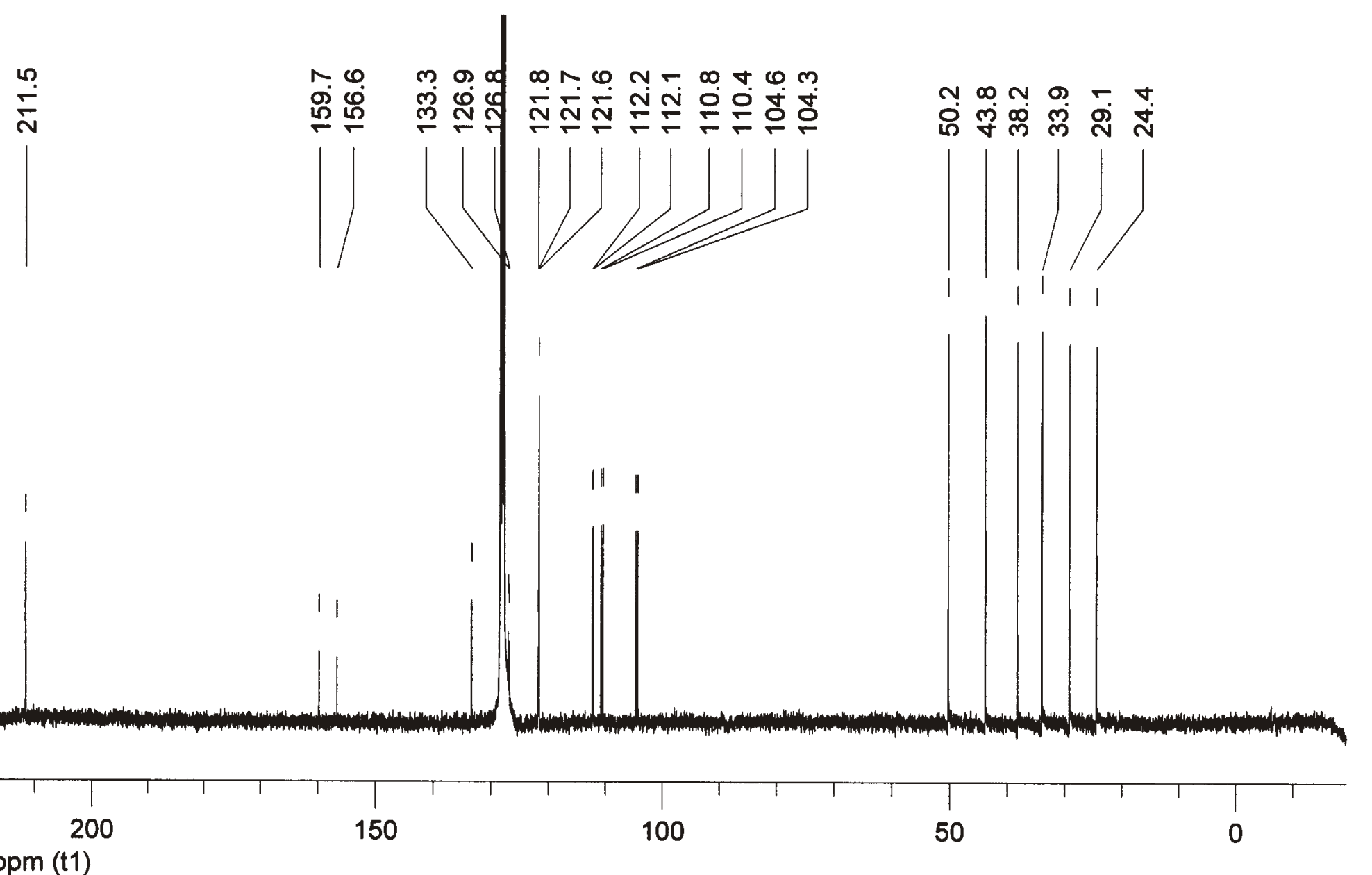




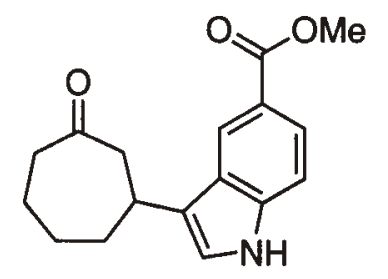

Table 3, Entry 8 3h
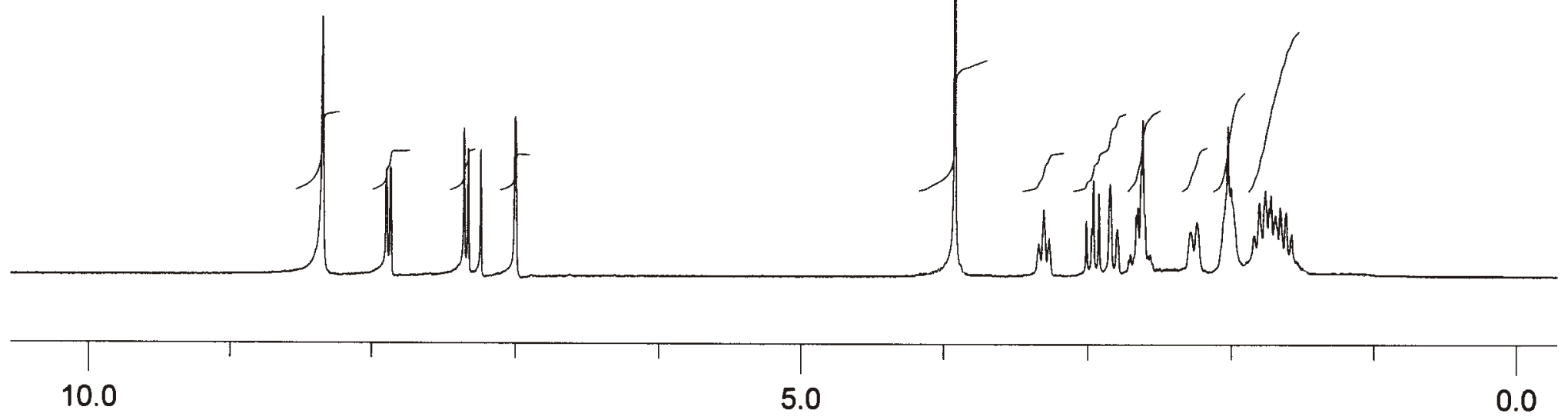

ppm (t1)

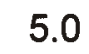

0.0
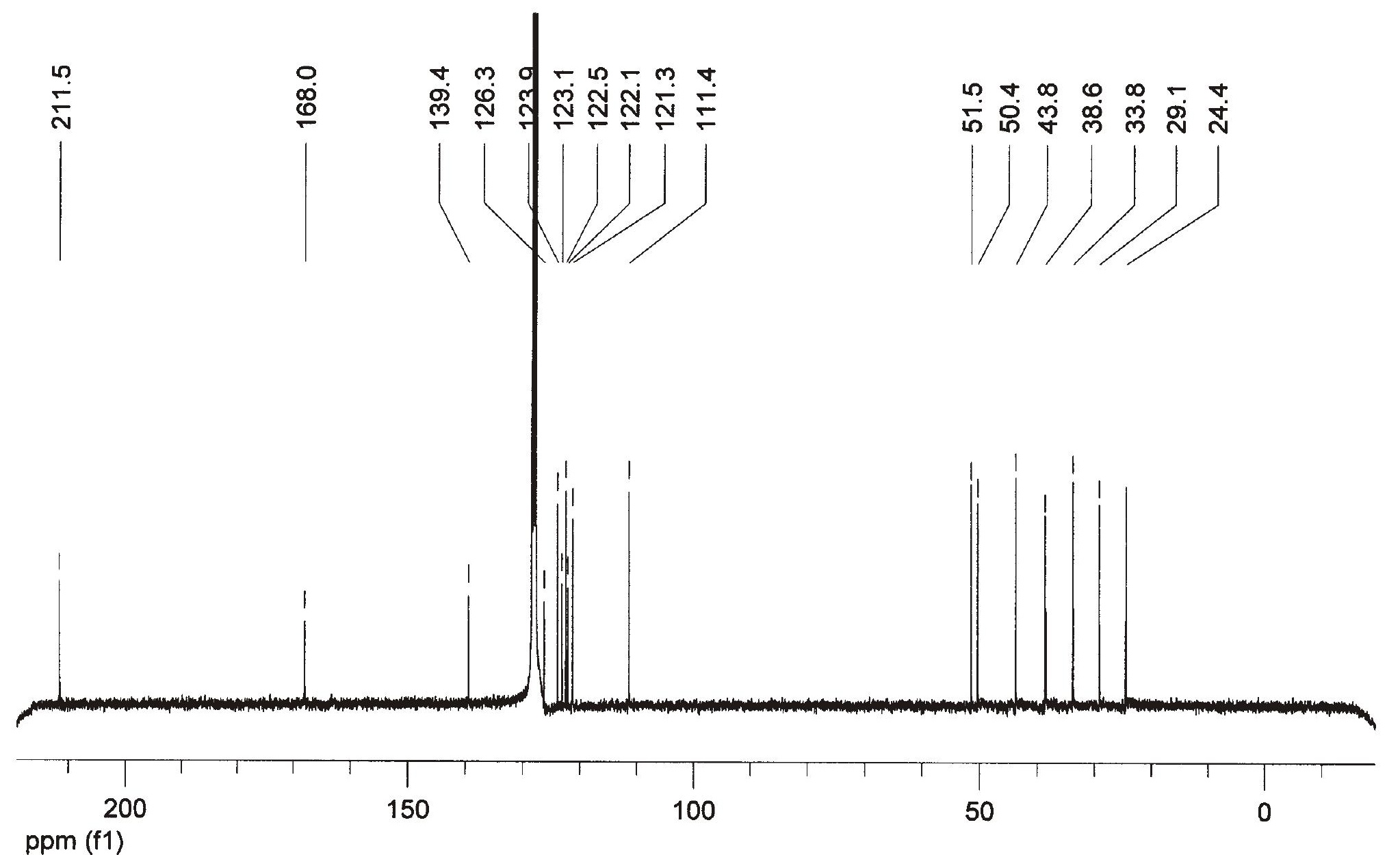

ppm (f1) 
<smiles>Cn1cc(C2CCCCC(=O)C2)c2ccccc21</smiles>

Table 4, Entry 2 $3 \mathrm{k}$

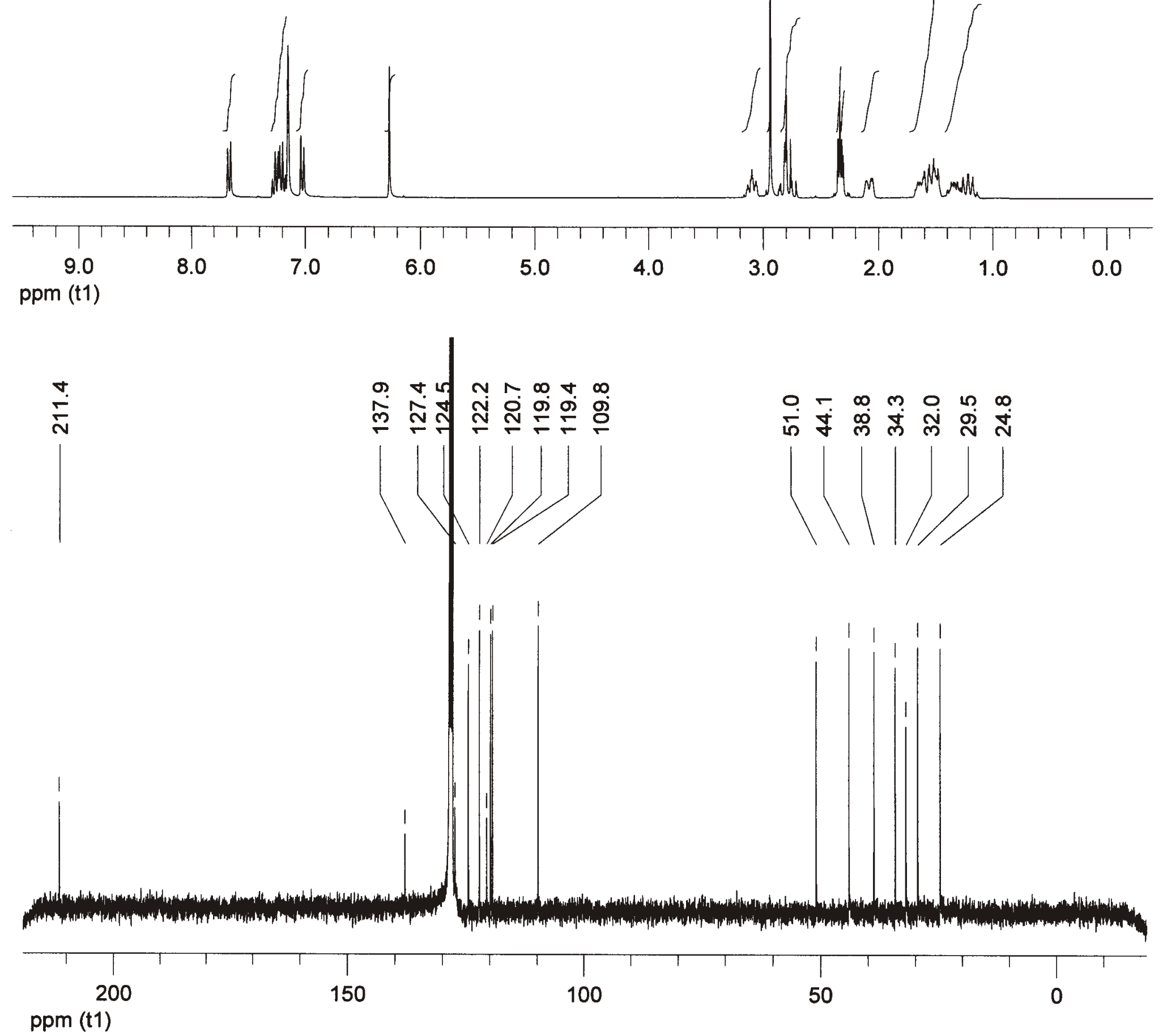


<smiles>C=CCn1cc(C2CCCCC(=O)C2)c2ccccc21</smiles>

Table 4, Entry 3 3।

$\stackrel{N}{\frac{N}{N}}$

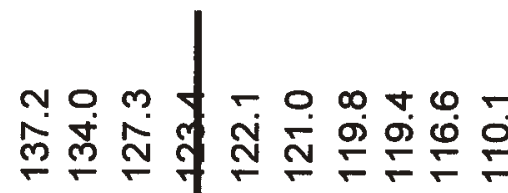

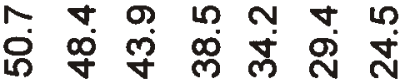
10.0 ppm (t1)
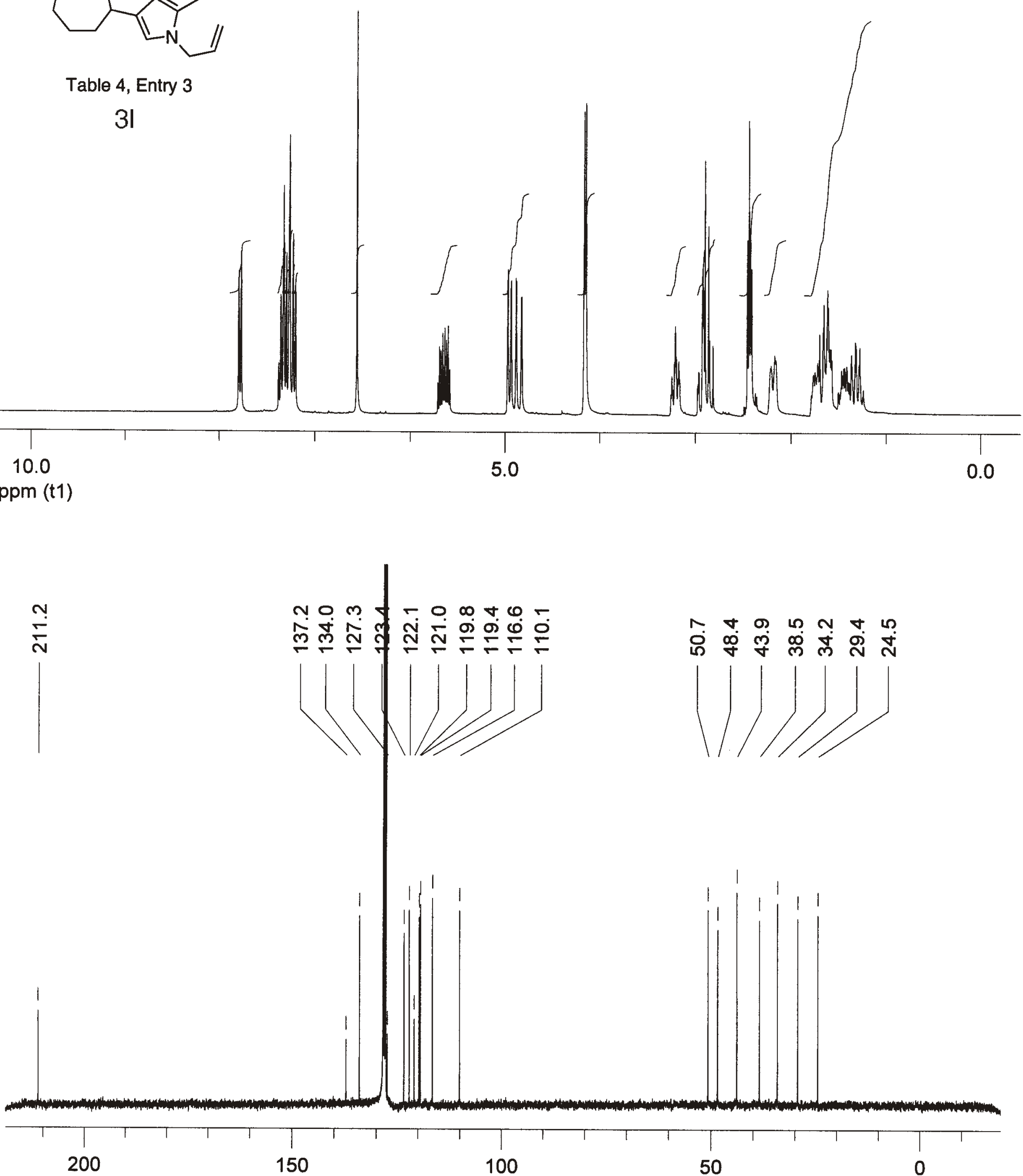

ppm (t1) 
<smiles>O=C1CCCCC(c2cn(Cc3ccccc3)c3ccccc23)C1</smiles>

Table 4, Entry 4 $3 \mathrm{~m}$

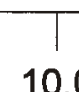

10.0

ppm (t1) $\stackrel{\circ}{\stackrel{ }{N}}$

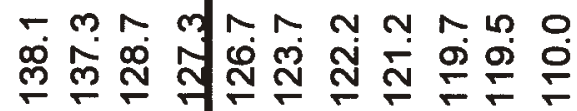
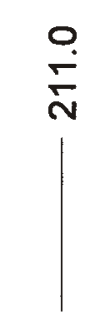

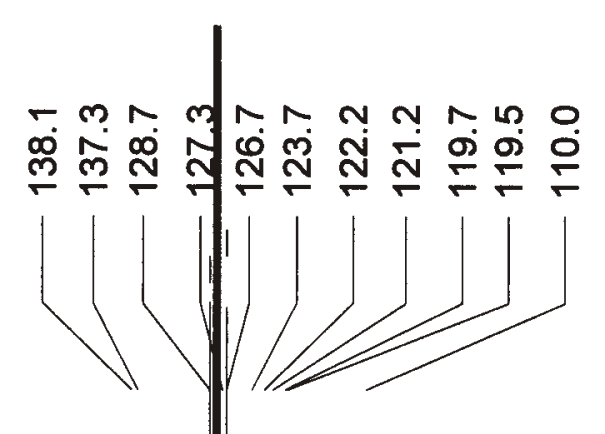

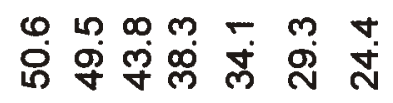
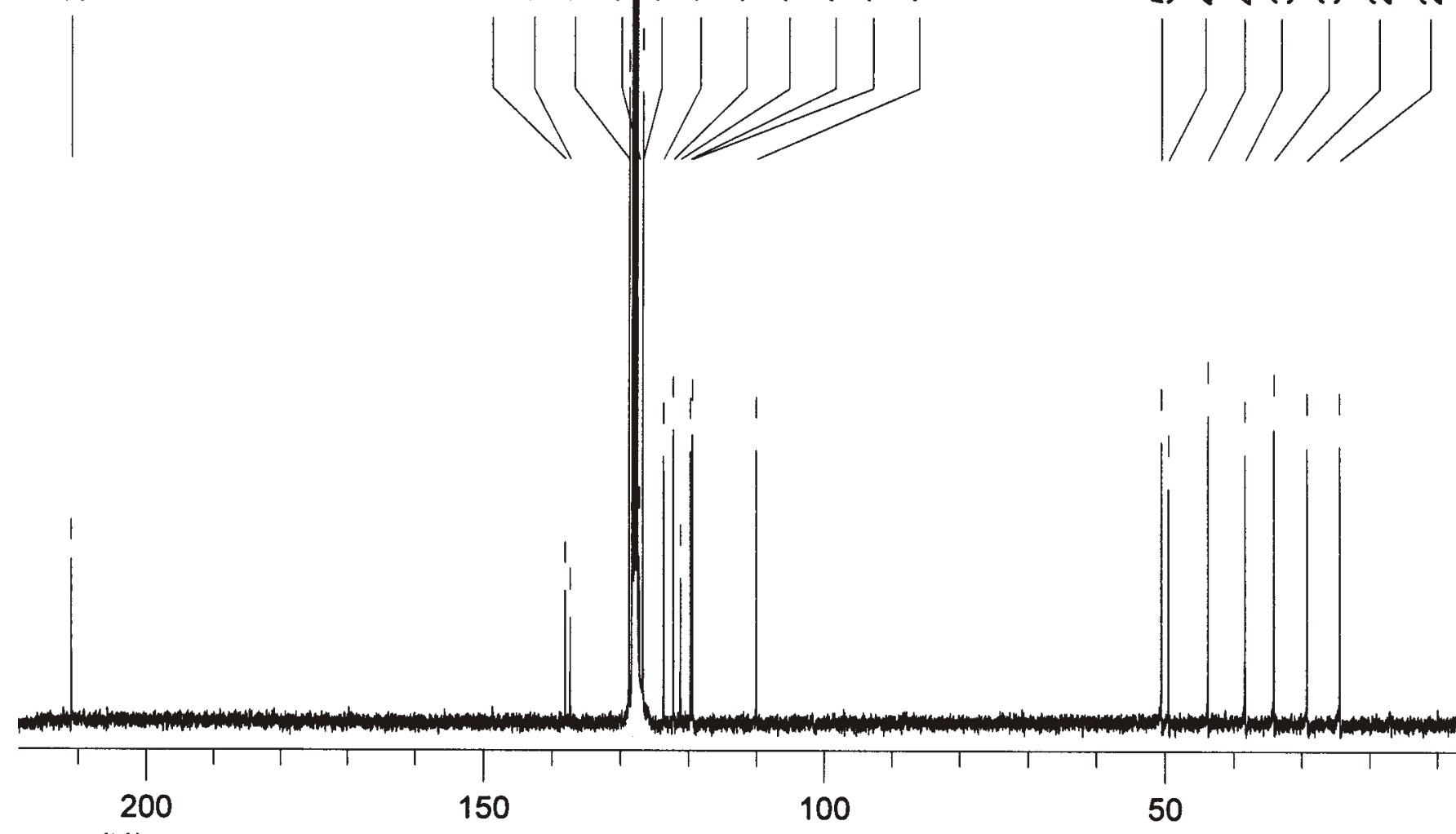

ppm (t1) 

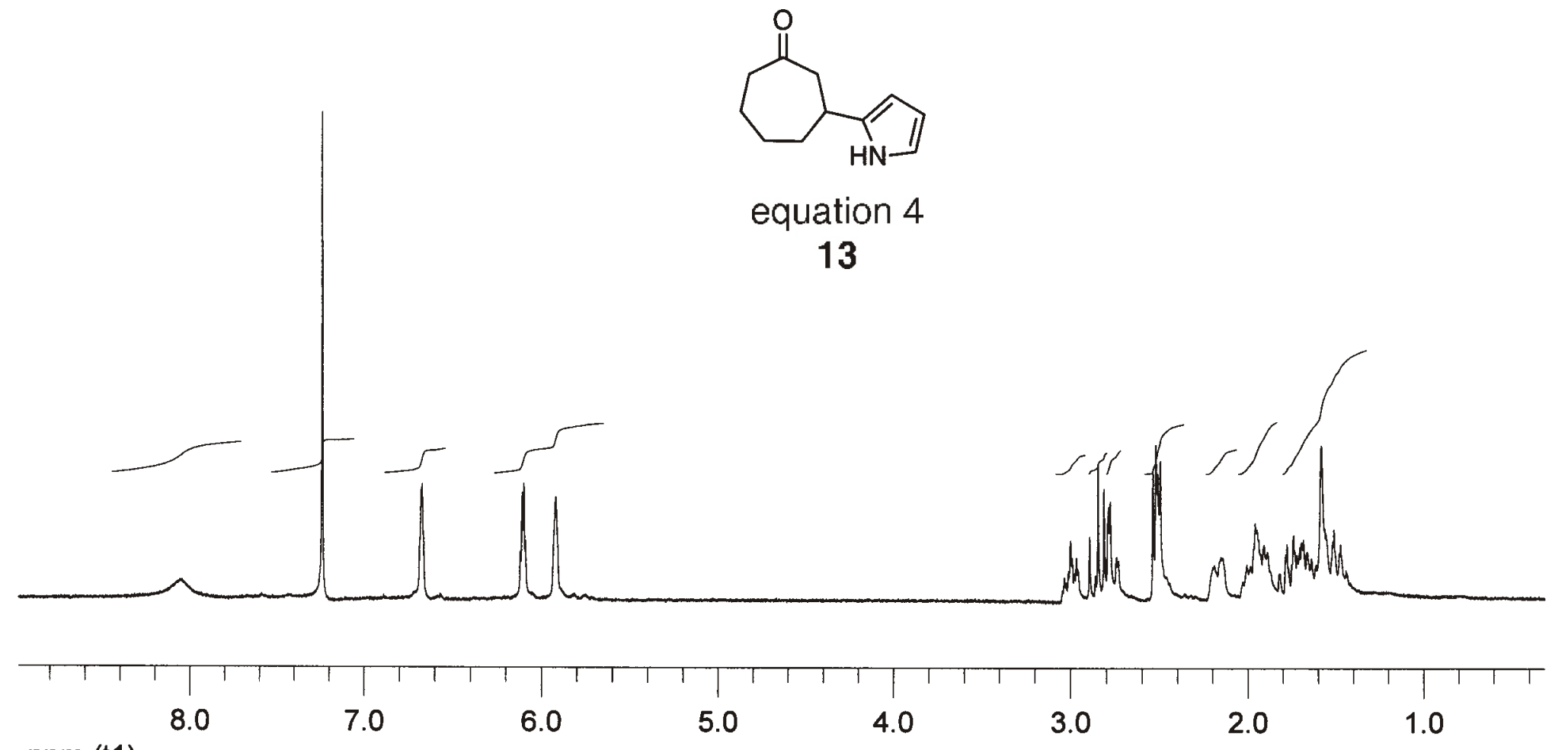

ppm (t1)

$$
\begin{aligned}
& \stackrel{\circ}{\stackrel{N}{N}} \\
& 1
\end{aligned}
$$

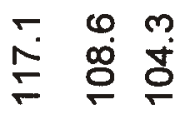

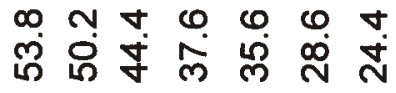
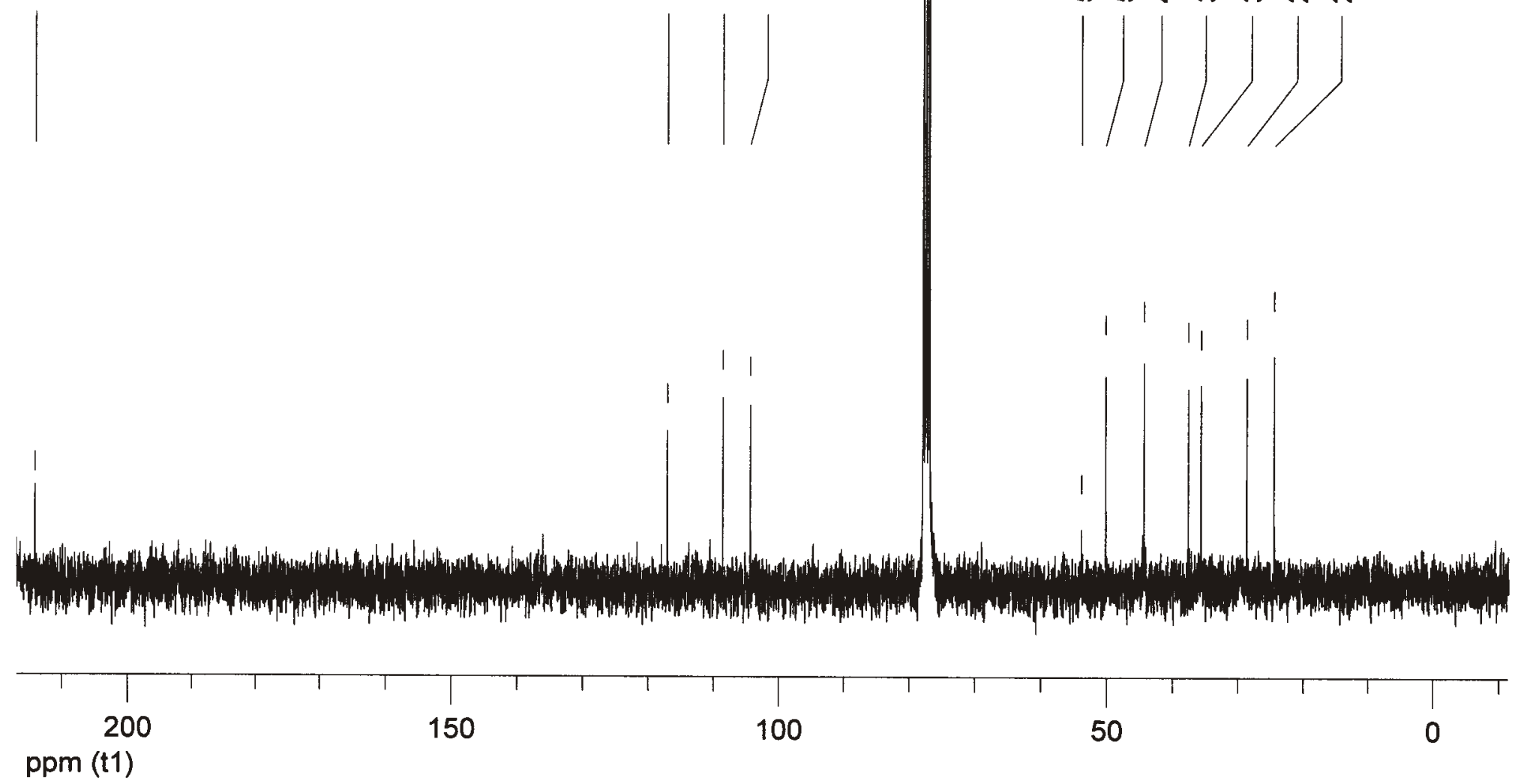